The Astrophysical Journal, 223:487-508, 1978 July 15

(C) 1978. The American Astronomical Society. All rights reserved. Printed in U.S.A.

\title{
ABUNDANCES IN GLOBULAR CLUSTER RED GIANTS. I. M3 AND M13
}

\author{
JUDITH G. COHEN \\ Kitt Peak National Observatory* \\ Received 1977 November 14; accepted 1978 January 24
}

\begin{abstract}
A detailed abundance analysis of five red giants in M13 and three in M3 has been performed using line-blanketed model atmospheres. Abundances have been determined for over 20 elements in each star. The mean Fe deficiency is -1.6 dex for M13 and -1.8 dex for M3, while M3 is marginally more metal-rich in Mg and Si than M13. Although all elements (with the probable exception of $\mathrm{Na}$ ) have the same abundance in each of the five M13 stars, considerable scatter exists for $\mathrm{Na}$ and $\mathrm{Ca}$ and perhaps other elements in the M3 stars. Fe and elements heavier than Fe have the same abundance in each of the three M3 red giants. It appears impossible to explain all the deduced scatter in M3 either by errors in the analysis or by convective mixing. Therefore, the primordial gas in M3 was not chemically homogeneous during the time interval when star formation of the currently observed M3 red giants took place.
\end{abstract}

Subject headings: clusters: globular - stars: abundances - stars: late-type

\section{INTRODUCTION}

The determination of abundances of the chemical elements in globular cluster red giants is of great interest, as they provide a key to understanding the chemical evolution of the Galaxy. We do not yet know whether the gas cloud out of which a given globular cluster condensed was initially chemically homogeneous, i.e., whether all the unevolved stars in a single globular cluster have identical abundances. Furthermore, while it has become apparent (Norris and Zinn 1977; Kraft, Carbon, and Butler 1978) that mixing, in the course of stellar evolution, has altered the $\mathrm{C}, \mathrm{N}$, and/or $\mathrm{O}$ abundances from their initial values, it is not yet clear whether mixing has affected the heavier elements. In addition, we seek to determine the nature of nucleosynthesis in the first few generations of stars (or in supermassive stars, or in the big bang) before the formation of the globular cluster stars we now see; we also seek evidence of how the buildup of elements from the most metal-poor globulars to those globulars with abundances close to solar proceeded. Clues to these questions can be found in an examination of the ratios of the abundances of various chemical elements. Tantalizing hints from the many analyses of metalpoor, late-type field stars (e.g., Pagel 1970; Hearnshaw 1972; Peterson 1978) indicate a general overdeficiency of the heavier elements as compared with the iron peak, with some evidence of an overdeficiency of odd elements suggestive of explosive nucleosynthesis as described in Arnett (1971).

The great improvement in detector technology over the past 10 years has made possible the search for answers to these questions. Using ordinary photo-

\footnotetext{
* Operated by the Association of Universities for Research in Astronomy, Inc., under contract with the National Science Foundation.
}

graphic emulsions, Helfer, Wallerstein, and Greenstein (1959) were barely able to reach the brightest giant in M13 and in M92. We have studied in detail five giants in M13 and three in M3. The second paper of this series will deal with M92 and M15, while the third will treat M71 and a few field $K$ giants. The final paper will analyze the $4300 \AA$ region of $\mathrm{CH}$ for one or two stars in each cluster except M3 and M15, where even the brightest giants are too faint to permit the obtaining of high-dispersion blue spectra.

In $\S$ II we describe the observational material and the measurement of equivalent widths, while $\S$ III discusses the derivation of abundances from the spectra and the necessary atomic data. The results of the detailed analysis are presented in $\S \mathrm{IV}$, and $\S \mathrm{V}$ contains a summary of the important conclusions. The radial velocities, interstellar lines, and $\mathrm{H} \alpha$ profiles in the globular giants are discussed in the Appendix.

\section{OBSERVATIONAL MATERIAL}

We have selected the brightest red giants observed by Cohen, Frogel, and Persson (1978, hereafter CFP) in each cluster as program stars. (Limitations on instrumental sensitivity force us to remain within 1 mag of the tip of the giant branch.) None of the program stars is a known variable according to the catalog of globular cluster variables (Hogg 1973). All observations have been made using the $4 \mathrm{~m}$ echelle spectrograph with the Singer camera, the 79 groove $\mathrm{mm}^{-1}$ echelle centered on the blaze, and the 226-1 cross disperser centered at $5500 \AA$. The resulting dispersion is $5.1 \AA \mathrm{mm}^{-1}$ at $5000 \AA$. Thus all the spectra are basically identical, except in the strength of the various metallic lines. Normally, the Singer camera was used with the transfer lens set at $\mathrm{f} / 2$ and with nitrogen-baked IIIa-J plates. Often the transfer lens was opened to $\mathrm{f} / 1.4$, and very rarely the nitrogen- 
TABLE 1

Plate Journal

\begin{tabular}{|c|c|c|c|c|c|c|c|c|c|c|c|c|}
\hline \multirow[b]{2}{*}{ Star } & \multicolumn{3}{|c|}{ Spectrum 1} & \multicolumn{3}{|c|}{ Spectrum 2} & \multicolumn{3}{|c|}{ Spectrum 3} & \multicolumn{3}{|c|}{ Spectrum 4} \\
\hline & number ${ }^{\star}$ & $t(\min )$ & weight & number* & $t(\min )$ & weight & number* & $t(\mathrm{~min})$ & weight & number* & $t(\min )$ & weight \\
\hline \multicolumn{13}{|l|}{$\underline{M 13}$} \\
\hline $\begin{array}{l}\text { BI } 40 \\
\text { IV }-25 \\
\text { I- } 13 \\
\text { III } 63 \\
\text { III }-73\end{array}$ & $\begin{array}{r}238 \\
259 \\
1161 \\
959 \\
1175\end{array}$ & $\begin{array}{r}60 \\
175 \\
100 \\
90 \\
103\end{array}$ & $\begin{array}{l}4 \\
3 \\
3 \\
2 \\
3\end{array}$ & $\begin{array}{r}244 \\
277 \\
1164 \\
1001 \\
1228\end{array}$ & $\begin{array}{r}60 \\
75 \\
90 \\
68 \\
139\end{array}$ & $\begin{array}{l}3 \\
5 \\
2 \\
4 \\
3\end{array}$ & $\begin{array}{r}276 \\
954 \\
1172 \\
1160\end{array}$ & $\begin{array}{r}60 \\
210 \\
150 \\
60\end{array}$ & $\begin{array}{l}5 \\
2 \\
3 \\
4\end{array}$ & $\begin{array}{c}960 \dagger \\
1232\end{array}$ & $\begin{array}{r}32 \\
152\end{array}$ & $\begin{array}{l}2 \\
4\end{array}$ \\
\hline \multicolumn{13}{|l|}{$\underline{\mathrm{M} 3}$} \\
\hline $\begin{array}{l}\text { VZ1397 } \\
\text { II } 46 \\
\text { III- } 28\end{array}$ & $\begin{array}{l}958 \\
243 \\
242\end{array}$ & $\begin{array}{l}94 \dagger \\
90 \\
90\end{array}$ & $\begin{array}{l}2 \\
2 \\
2\end{array}$ & $\begin{array}{r}1007 \\
275 \\
957\end{array}$ & $\begin{array}{l}120 \\
100 \\
92 \dagger\end{array}$ & $\begin{array}{l}5 \\
4 \\
2\end{array}$ & $\begin{array}{l}1174 \\
1171 \\
1163\end{array}$ & $\begin{array}{l}140 \\
135 \\
140\end{array}$ & $\begin{array}{l}4 \\
4 \\
4\end{array}$ & 1167 & 159 & 4 \\
\hline
\end{tabular}

*all numbers are in the SI series (Singer image tube, $4 \mathrm{~m}$ echelle)

tbaked II a-O emulsion

baked IIa-O emulsion was used. The projected slit width was $38 \mu \mathrm{m}$; on nights of bad seeing this was increased to $42 \mu \mathrm{m}$. The individual echelle orders are between 0.2 and $0.3 \mathrm{~mm}$ wide-rarely wider except for the brightest stars. At least two and usually three spectra were obtained for each star. The spectra available for each star are listed in Table 1, where the exposure time (in minutes) is also listed.

The spectra were traced using the PDS digital microphotometer in a raster pattern which included echelle orders 34 (centered at $6750 \AA$ ) through 49 $(4700 \AA)$. The sensitometer calibration plates, exposed and developed simultaneously with the spectra, were also traced with the PDS. Sensitometer plates were taken for each exposure-rarely every second exposure and always each time spectra were developed. The location of the orders, conversion to intensity, and production of a plot of intensity along each order were done with the standard Kitt Peak National Observatory echelle reduction software as described in Cohen (1976a). The typical full width at half-maximum of weak lines was approximately $0.35 \AA$.

A single spectrum of one of the coolest stars was selected as the master spectrum for line identifications, which were made using Moore, Minnaert, and Houtgast (1966, hereafter MMH), Griffin (1968), Griffin (1969), and Mäckle et al. (1975a) as guides. Because of the larger metal deficiency in M3 and M13, the determination of the continuum level was not a problem until below $5000 \AA$. Relatively few lines were measured below that wavelength because of increased blending of lines, problems in locating the continuum, and increasing weakness of the spectra toward the blue. Lines with adjacent blends of comparable or greater strength closer than $0.5 \AA$ were considered too blended to measure.

For each order, the dispersion was determined and assumed constant over the order (this assumption is correct to within $1 \%)$. Equivalent widths $\left(W_{\lambda}\right)$ were then measured using a triangle approximation for the set of lines defined as unblended on the master tracing. The spectra were measured in random order, and all measuring and line identifications were done by the author. After all the plates for a star had been measured the spectra were examined visually and weights were assigned to each spectrum. These weights are listed in Table 1. A weighted average of the measured equivalent widths for each line was then calculated, with half the appropriate weight given to measurements that were uncertain because of serious blending, plate defects, asymmetric line profiles, or uncertainty of the continuum. The weighted $W_{\lambda}$ are listed for each star in Table 2. The values of $W_{\lambda}$ for lines with a total weight less than $50 \%$ of the maximum weight for that star are indicated by a colon. The total weight for each individual line is available from the author upon request. It is difficult to assess the accuracy of these measurements. For lines with $W_{\lambda}<50 \mathrm{~m} \AA$, the error is $\pm 10 \mathrm{~m} \AA$. For stronger features, a reasonable estimate of the error is $\pm 20 \%$. However, as can be seen by perusing Table 2 , the relative error in comparing $W_{\lambda}$ for a given line, as measured in several stars, is less than the errors quoted above for the absolute value of $W_{\lambda}$ for lines with high weights (i.e., unblended lines measured on all the spectra of a given star) toward the center of the echelle orders. Absence of values for $W_{\lambda}$ in Table 2 does not necessarily imply that the line was not detected $\left(W_{\lambda} \leqslant 20 \mathrm{~m} \AA\right)$; the echelle centering determines the exact wavelengths included on the plate (i.e., the free spectral range is larger than the image-tube diameter), and about $40 \%$ of the spectrum between 4700 and $6700 \AA$ is missing on any single exposure. In particular, we obtained one beautiful spectrum for VZ 1397, where the echelle was set accidentally toward shorter wavelengths than the normal blaze center; hence many lines are seen only in this star.

\section{ATOMIC-LINE PARAMETERS}

Our ultimate goal is a differential analysis of the globular cluster giants with respect to the Sun. We have adopted the HSRA solar model (Gingerich $e t$ al. 1971) with a microturbulent velocity of $1 \mathrm{~km} \mathrm{~s}^{-1}$ (Smith, Testerman, and Evans 1976; Furenlid and 


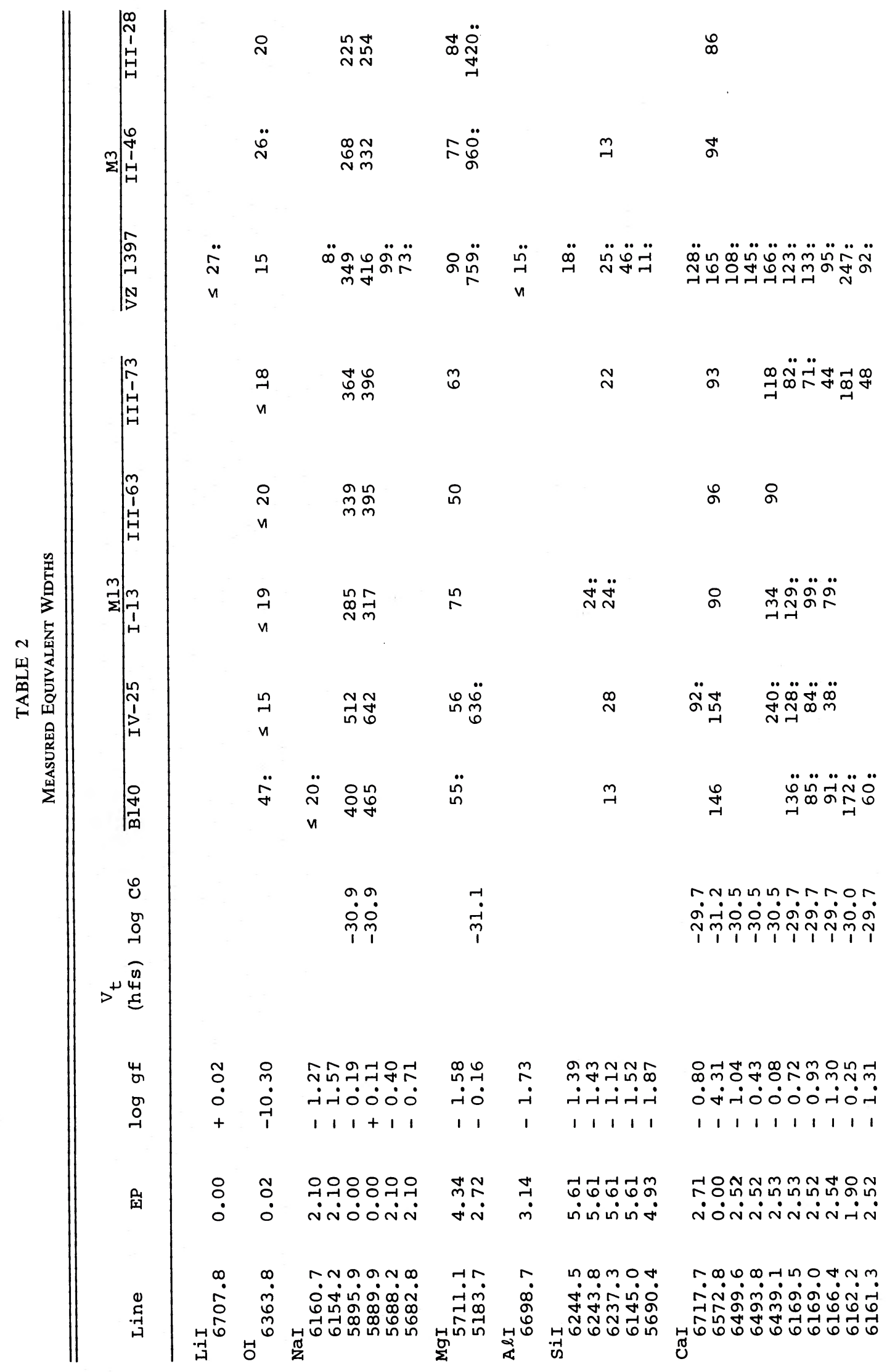




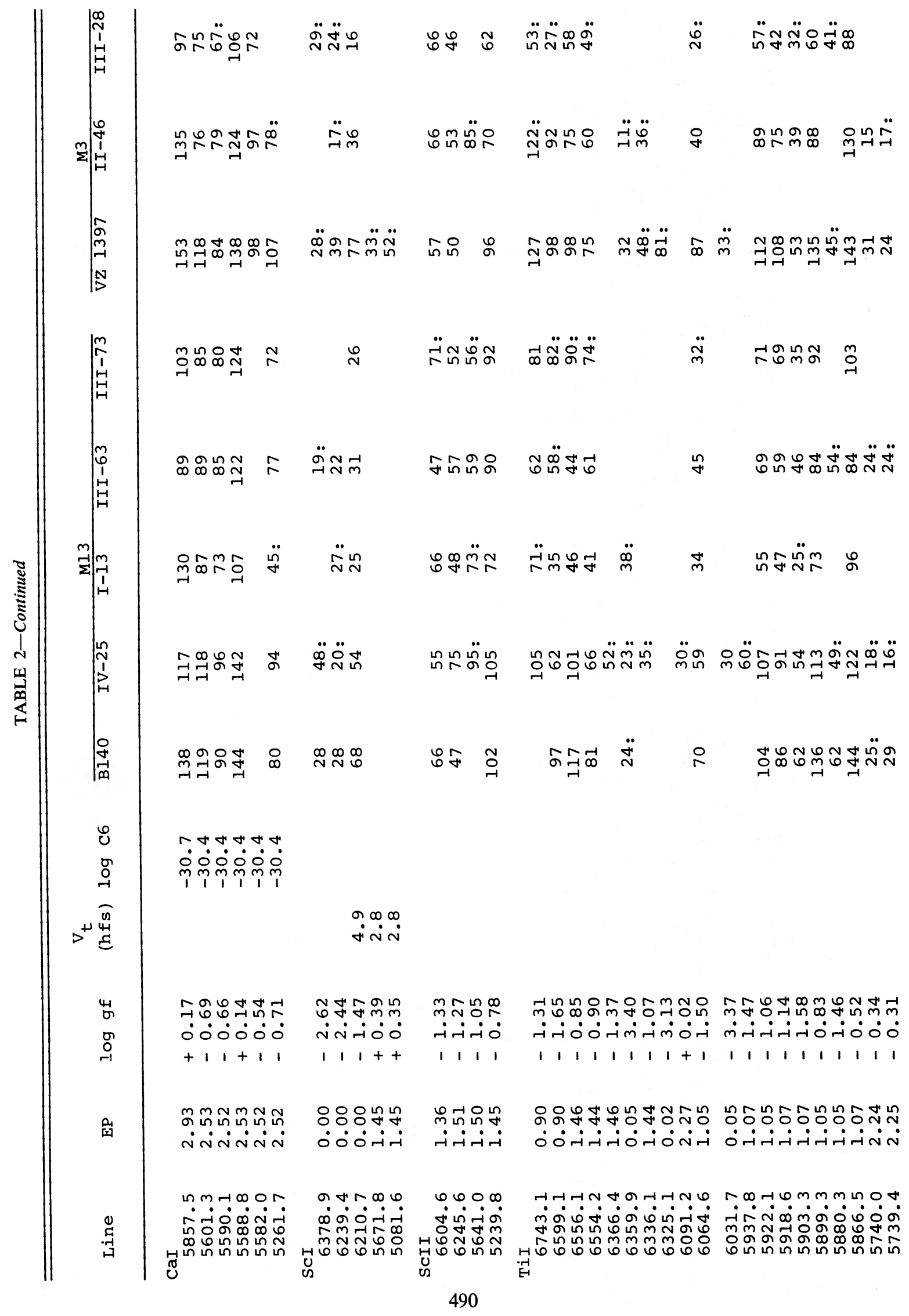




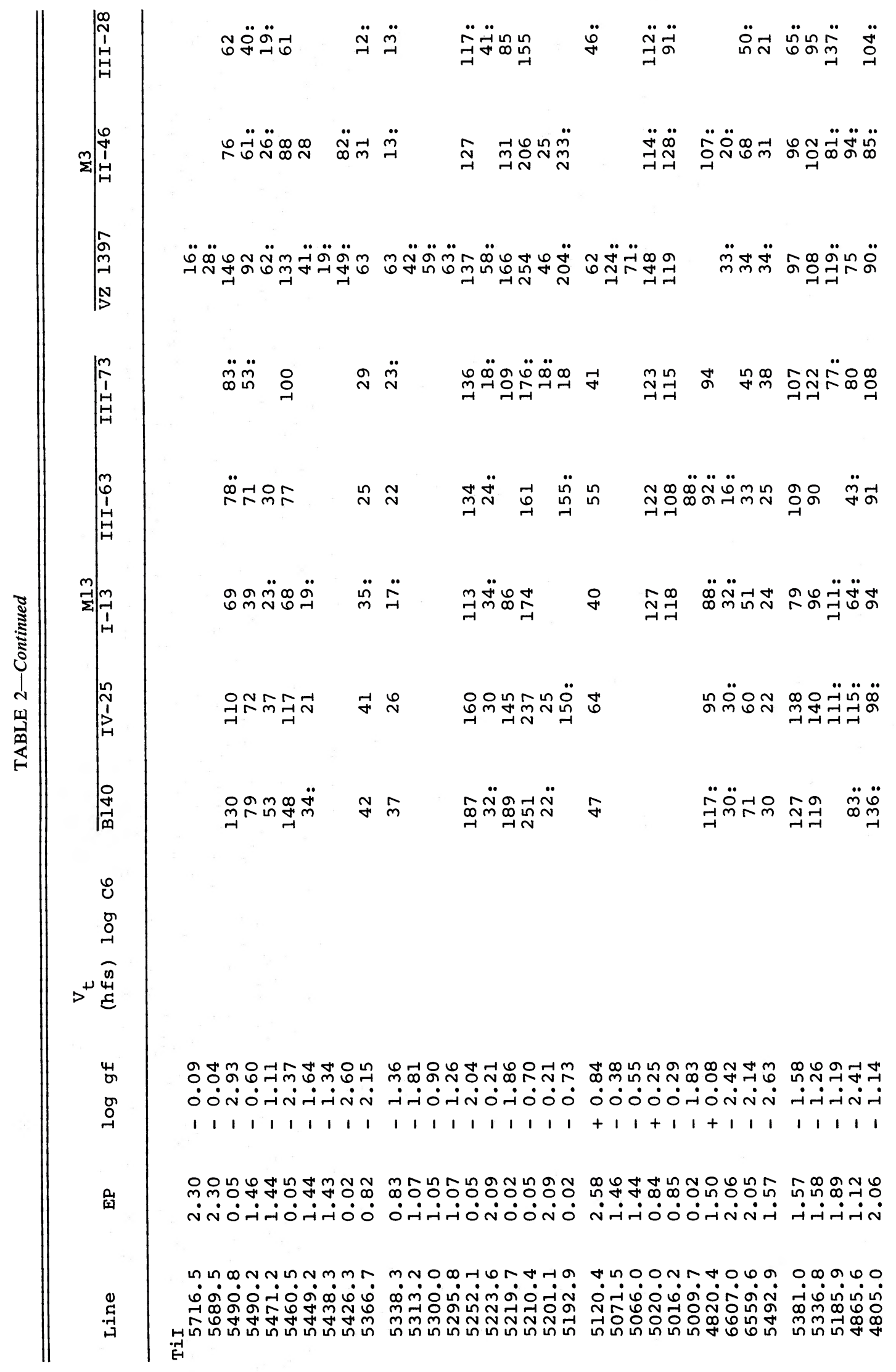

491 


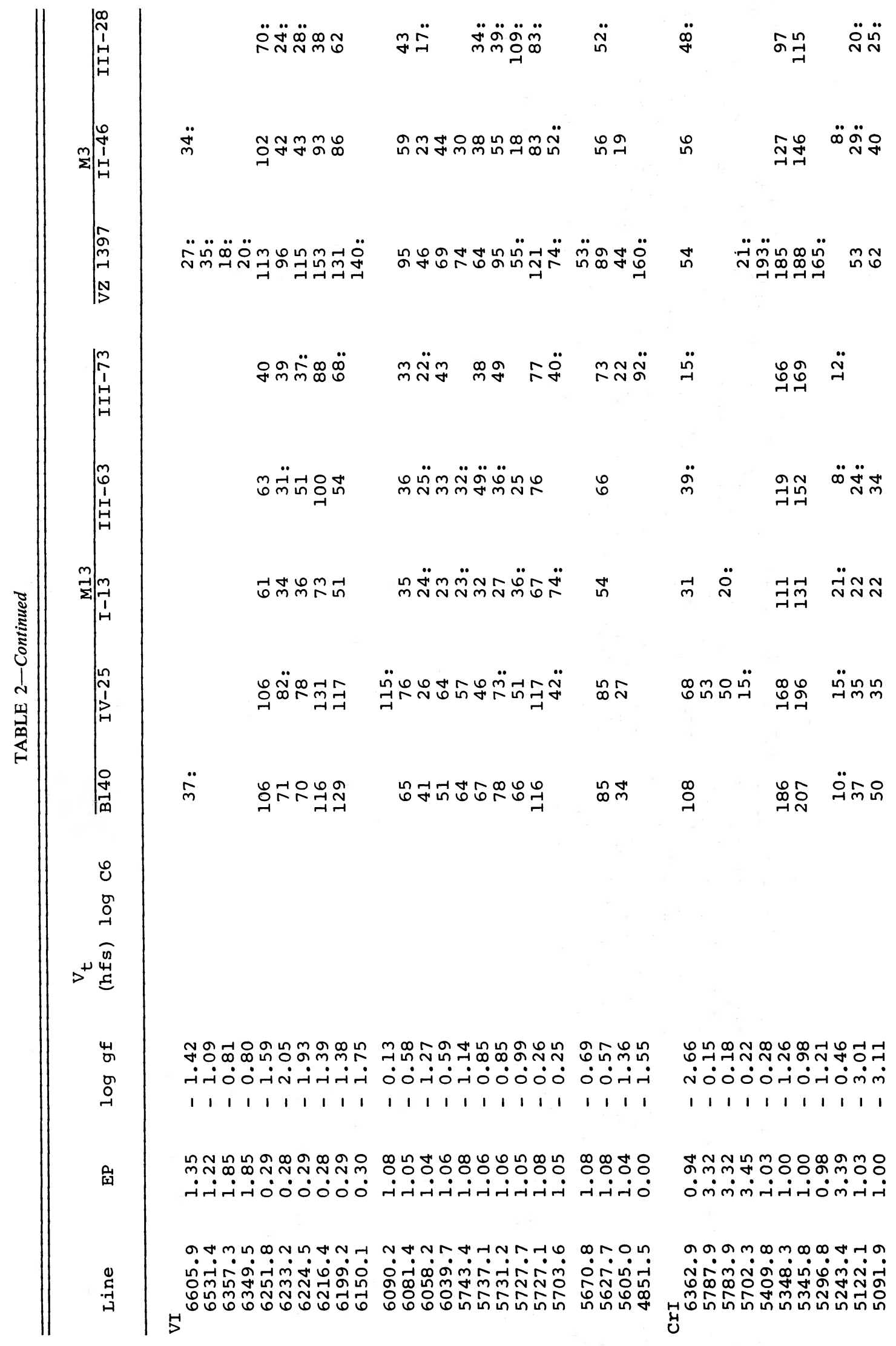




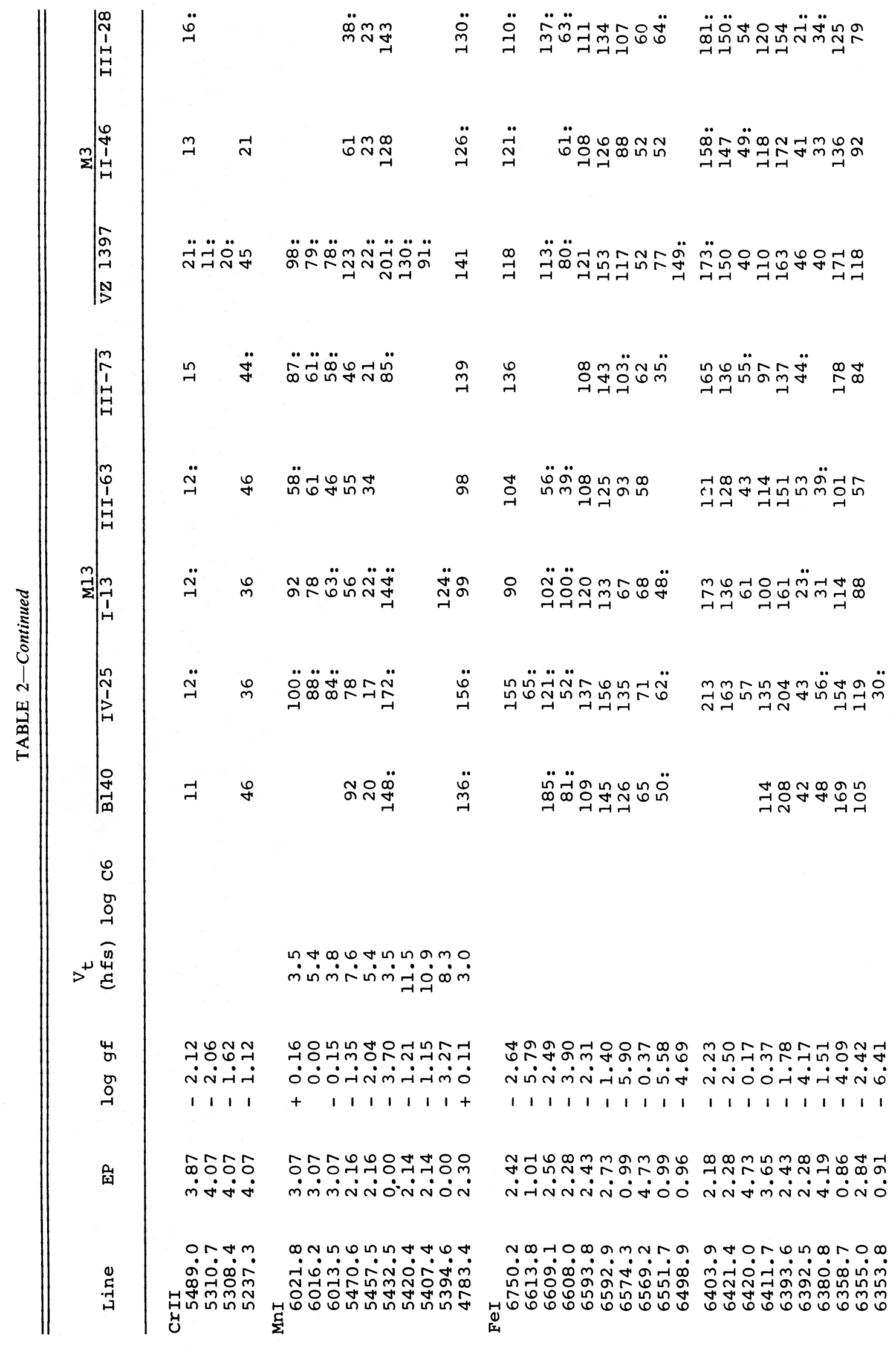




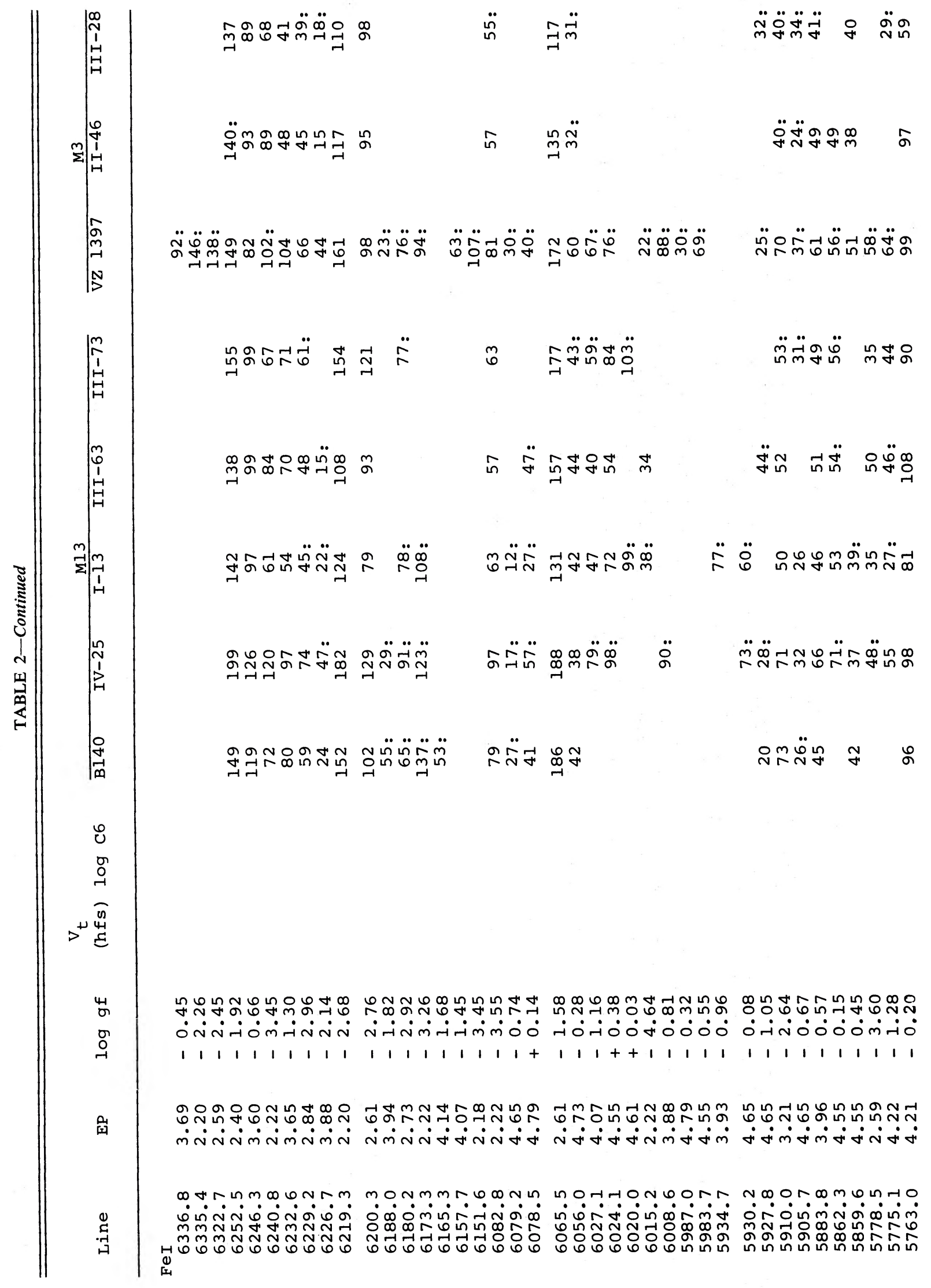




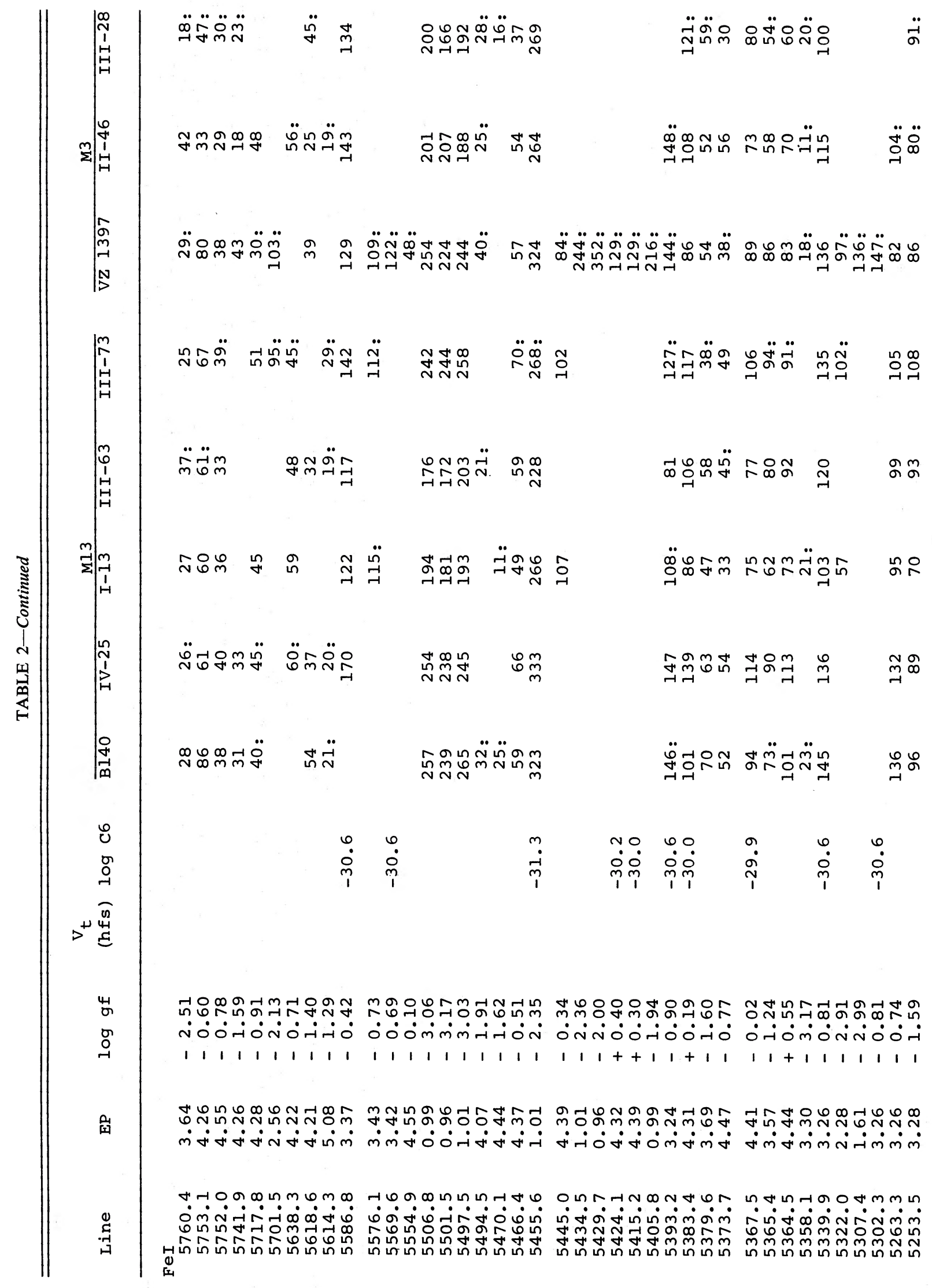

495 


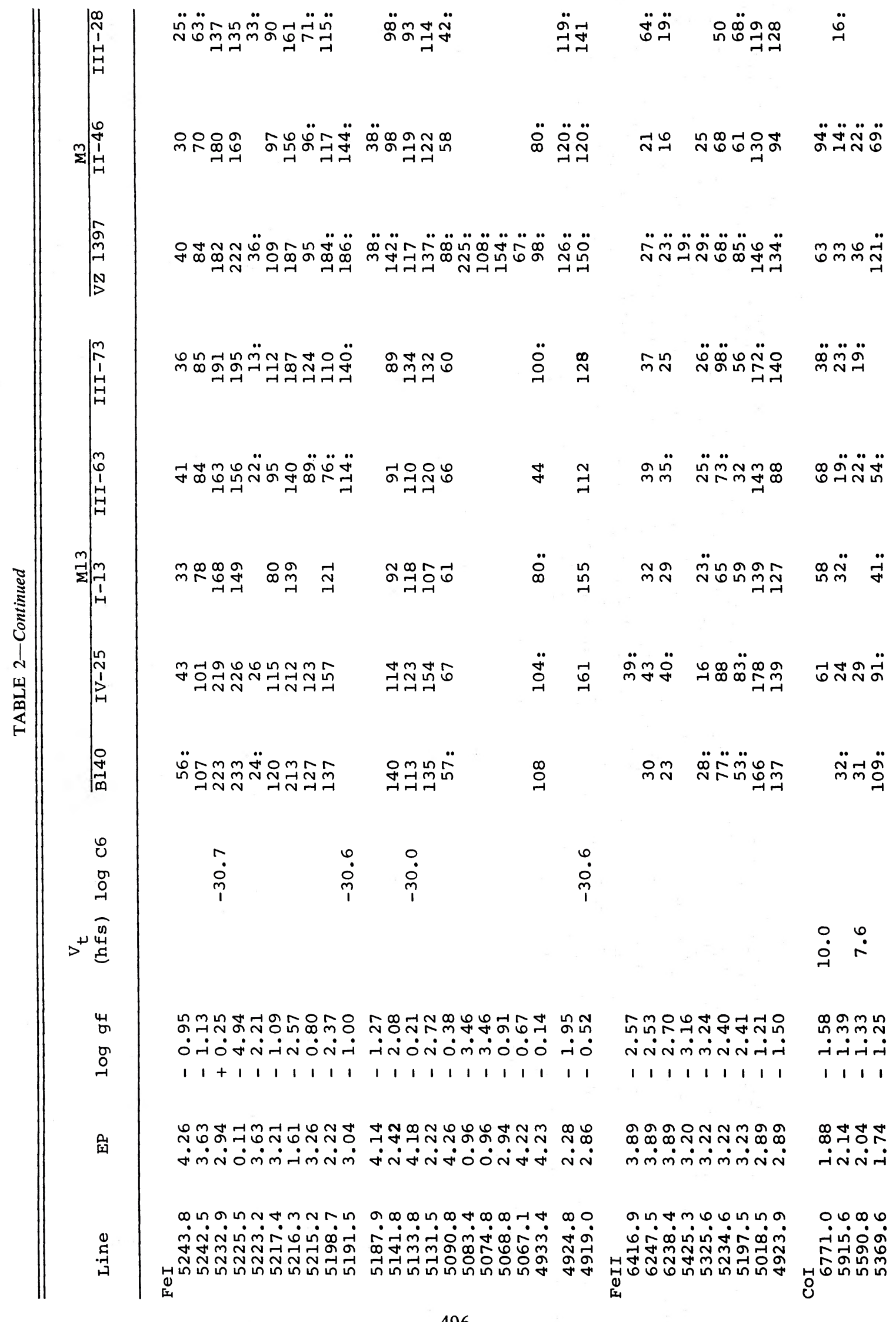

496 


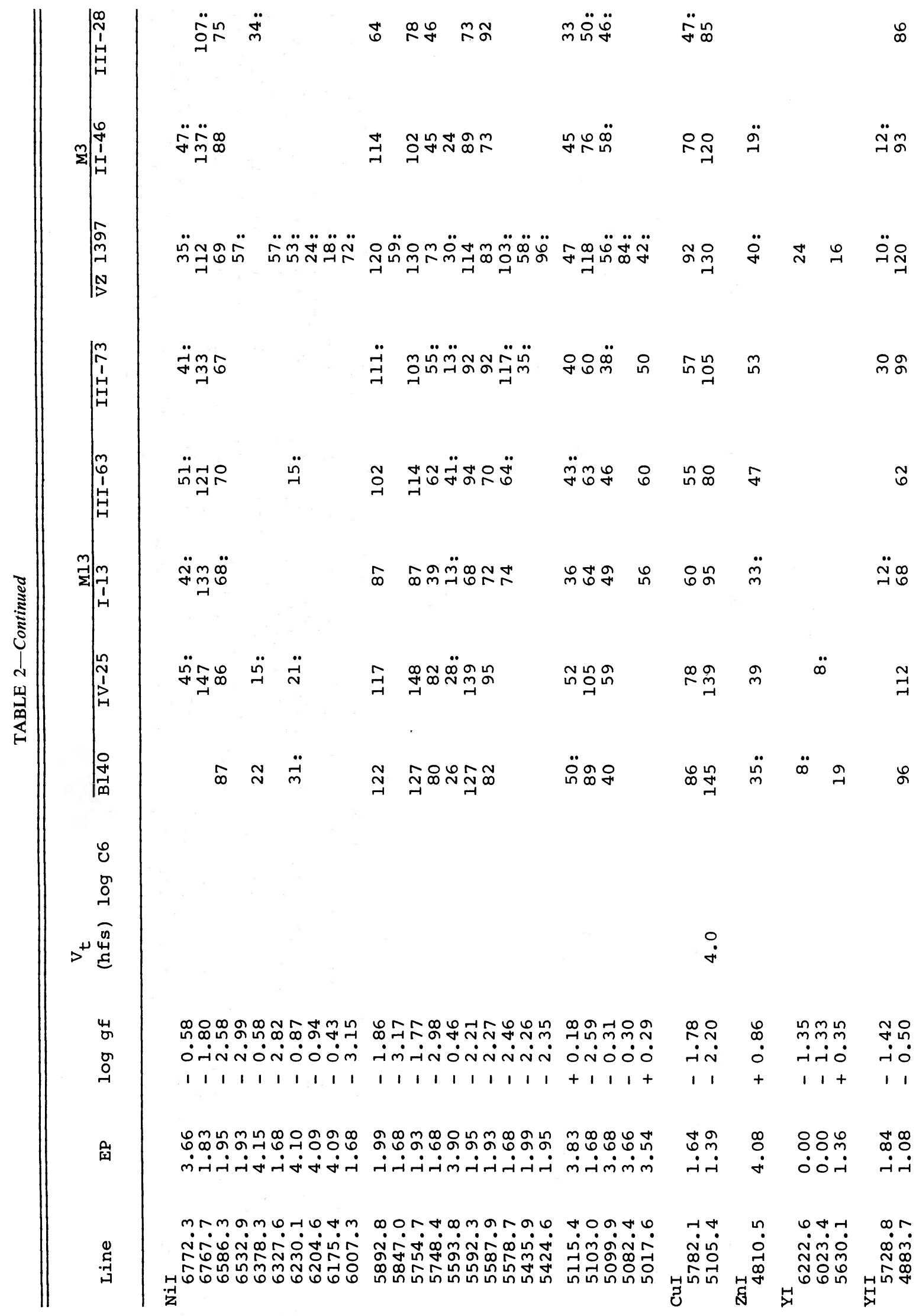

497 


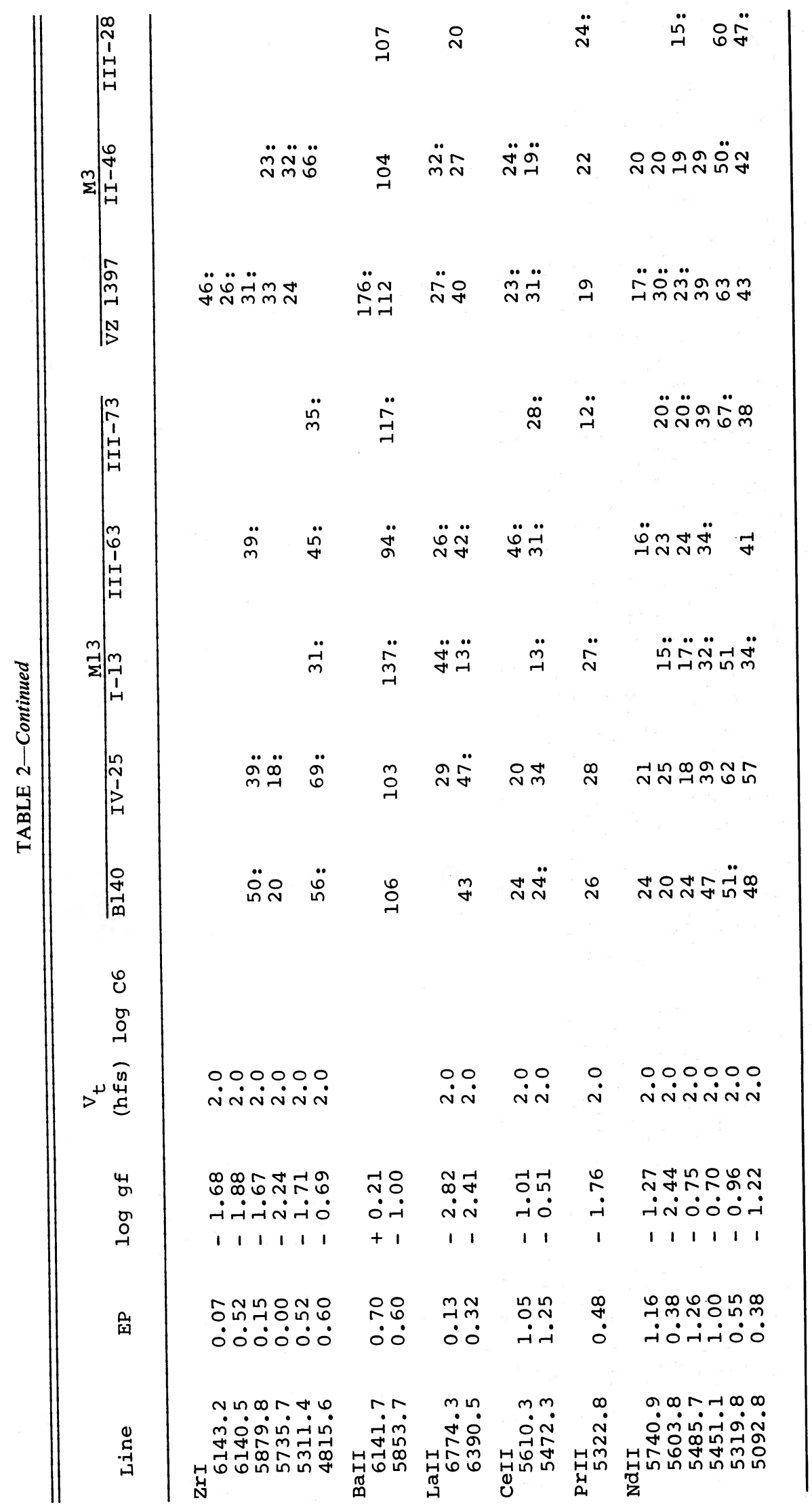


Condal 1976) for the Sun. For the red giants, we have used the grid of models computed with the code ATLAS6 (Kurucz 1970, 1978; Kurucz, Peytremann, and Avrett 1974; Kurucz and Peytremann 1975) described in CFP. These are local thermodynamic equilibrium (LTE) models, with opacity from a large list of atomic (but no molecular) lines included. Rayleigh scattering is explicitly included in the opacity calculation in the ATLAS and abundance analysis codes. CFP have demonstrated that $V-K$ is an excellent indicator of effective temperature $T_{\text {eff }}$, and we have used the $T_{\text {eff }}$ determinations from infrared observations given by them (called $T_{\text {eff }}{ }^{\circ}$ ) for the globular cluster giants. We have also used surface gravities $g^{0}$ calculated from the known distance moduli of the globular clusters, from the observed bolometric corrections, and from an assumed mass of $0.8 M_{\odot}$ as described in CFP. The adopted values are listed in Table 3. We expect the values of $T_{\text {eff }}$ given by CFP to be accurate to $\pm 100 \mathrm{~K}$ on an absolute scale and to $\pm 50 \mathrm{~K}$ in comparing giants in M3 and M13. The maximum possible error in the surface gravity, when one allows both for uncertainties in the unknown mass of the red giants and for possible problems in the distance moduli, is a factor of 2 or \pm 0.3 in $\log g$.

Given a model atmosphere, microturbulent velocity $V_{t}$, transition probability $g f$, possible hyperfine or isotopic broadening, and damping constant, the computer code WIDTH (Kurucz 1970) can determine for each observed line the abundance of the ion which produces the observed $W_{\lambda}$. The most serious obstacle remaining is the collection of a set of suitable atomicline parameters. We require only relative $g f$-values, since we can determine the fictitious solar abundance for an ion corresponding to our choice of absolute scale for the $g f$-values by using the observed solar $W_{\lambda}$ from $\mathrm{MMH}$ with our absolute $g f$ scale through our solar model in WIDTH. The mean abundance of the observed set of lines for any ion is the fictitious solar

TABLE 3

Model Atmosphere Parameters

\begin{tabular}{lccccc}
\hline \hline & & & & $V_{t}$ & \\
Star & $T_{\text {eff }}^{\circ}$ & $\log g^{\circ}$ & $Z / Z_{\odot}$ & $\left(\mathrm{km}^{-1}\right)$ & Note \\
\hline MI3 & & & & & \\
B140 & 4000 & 0.5 & 0.03 & 2.0 & 1 \\
IV-25 & 4000 & 0.5 & 0.03 & 2.2 & 2 \\
I-13 & 4250 & 0.9 & 0.03 & 2.0 & 3 \\
III-63 & 4200 & 0.7 & 0.03 & 1.5 & 2 \\
III-73 & 4300 & 0.8 & 0.03 & 2.0 & 2 \\
& & & & & \\
M3 & & & & & 2 \\
VZ1397 & 4000 & 0.6 & 0.03 & 2.0 & 2 \\
II-46 & 4000 & 0.6 & 0.03 & 2.0 & 2.0 \\
III-28 & 4100 & 0.7 & 0.03 & 2 & \\
\hline
\end{tabular}

NOTES: 1) $T_{\text {eff }}^{\circ}$ and $\log g^{\circ}$ assumed as that of IV-25 because of similar $V$ magnitudes.

2) Tㅇff and $\log g^{\circ}$ from CFP.

3) $T_{\text {eff }}^{\circ}$ and $\log g^{\circ}$ from unpublished infrared photometry by Frogel and Persson (1977). abundance of that ion. Such fictitious abundances have been derived using only the lines of each ion observed in the globular cluster giants, except for $Y_{I}, Y_{\text {II, }}$ $\mathrm{Zr}$ I, Ce II, Pr II, and Nd II. For these ions, very few lines are observed in the globular giants, and the $W_{\lambda}$ in the Sun tend to be very small $\left(W_{\lambda} \leq 5 \mathrm{~m} \AA\right)$ for the observed lines. We have therefore assumed that, for these ions, the Corliss and Bozman (1962, hereafter CB) relative $g f$-values are correct; we have searched $\mathrm{MMH}$ for the strongest unblended lines of each ion with $\lambda>4500 \AA$ and have used these lines to determine the fictitious solar abundance. The same procedure was carried out for $\mathrm{Sc} \mathrm{I}$, using the relative $g f$-values of Wiese and Fuhr (1975). The situation is even worse for $\mathrm{Zr}$ I in that there are no reasonably strong solar lines; we have had to assume that the CB $g f$ values for $\mathrm{Zr}$ I and $\mathrm{Zr}$ II are on the same absolute scale using the fictitious solar abundance for $\mathrm{Zr}$ II to normalize our $\mathrm{Zr}$ I results. For $\mathrm{VI}$, we have assumed the relative CB $g f$-values to be correct and have derived the fictitious solar abundance from the mean of the stronger $\mathrm{V} I$ lines observed in the globular giants and the Sun. The weaker V I lines have $W_{\lambda}$ too small in the Sun to allow derivation of reliable relative $g f-$ values through the solar model. Because of suspected errors in the CB $g f$-values dependent on excitation potential (Bridges and Wiese 1970), we preferred not to use them as relative $g f$-values for any ion unless it became necessary. Instead, for $\mathrm{Ca}$ I and $\mathrm{Na}$ I, we have used the $g f$-values and damping constants of Holweger (1971, 1972). For other ions, we have assumed a damping constant 10 times the classical value, and have used the observed solar $W_{\lambda}$ from MMH together with our solar model to derive relative $g f$-values. For the $\mathrm{Mg}_{\mathrm{I}}$ line at $5183 \AA$ and for the strongest $\mathrm{Fe}_{\mathrm{I}}$ lines $\left[W_{\lambda}\right.$ (Sun) $\left.\geq 150 \mathrm{~m} \AA\right]$, damping constants were computed using the prescription of Holweger (1972) for the collisional damping constant $\mathrm{C}_{6}$, since $10 \Gamma_{\mathrm{cl}}$ gave very unsatisfactory results, especially for the strongest $\mathrm{Fe} \mathrm{I}$ lines. Accurate damping constants are a real problem (Foy 1972); but since we have used relatively weak lines in the Sun and globular giants, our results do not seriously depend on the choice of $\Gamma$.

To correct for broadening due to hyperfine structure (hfs) or isotopic splitting, we inspected the KPNO solar atlas (Brault and Testerman 1977) near the observed Mn I lines. J. Brault kindly made available an FTS spectrum of Co I by which the hyperfine structure of the lines we observed could be checked. Hyperfine broadening was allowed for by artificially increasing the value of $V_{t}$ for each observed line with hfs. These values of $V_{t}$ were derived from the observed full width at half-maximum of the solar lines (with a solar $V_{t}$ of $1 \mathrm{~km} \mathrm{~s}^{-1}$ ) as compared with the instrumental profile and with solar lines that have negligible hfs. The relative $g f$-values of CB were assumed to be correct for Mn I and Co I; by using the corresponding $V_{t}$ for each line, a dispersion of \pm 0.11 was obtained about the mean fictitious solar Mn I abundance deduced from the $10 \mathrm{Mn}$ I lines observed in the Sun and in the globular cluster giants. As only four Co I 
lines were observed in the globular giants and as the $W_{\lambda}$ (Sun) given in MMH for those weak lines are too low, we have adopted the solar abundance for Co I derived from many solar lines by Holweger and Oertel (1971) using the CB $g f$-values and appropriate hfs corrections. The $\mathrm{V}$ I lines did not show enough broadening in the solar atlas to cause us to worry about hfs.

Hyperfine corrections were also made for lines of $\mathrm{Cu}$ I and $\mathrm{Sc} \mathrm{I}$ by adjusting the microturbulent velocity based on the observed solar full width at half-maximum for the appropriate lines in the KPNO solar atlas. Although the rare earths are known to have hyperfine structure (see the references in Mäckle et al. 1975b), much of this is due to isotopic splitting, and we have no information regarding the isotope distribution in the globular giants. Furthermore, the rare-earth lines were too weak to permit determination of their solar profiles and were also quite weak in the giants of M3 and M13. We have therefore arbitrarily introduced an extra microturbulence of $2 \mathrm{~km} \mathrm{~s}^{-1}$ into the elements heavier than yttrium-except for $\mathrm{Ba}$ II, where inspection of the solar atlas showed no extra broadening for the lines we used. The maximum change for the rareearth abundances was a decrease of 0.07 in the log (abundance of rare earth/H) - subsequently denoted by 0.07 dex-when such a broadening was introduced.

The choice of atomic parameters, whose values are listed in columns (2)-(5) of Table 2, has been made in accordance with the best available data as described in detail above. Although many may be slightly incorrect, we have acted to minimize any errors in abundances resulting from incorrect atomic parameters, since we have applied them uniformly to the Sun and to all the globular giants, and since the metal deficiency of M3 and M13 is such that most lines are of comparable strength in the globulars and in the Sun. We believe that errors resulting from our choice of $g f$ values, hfs, and damping constants should be not more than 0.1 dex on an absolute scale-except for Sc $\mathrm{I}$ and $\mathrm{Co} \mathrm{I}$, where an error of $0.15 \mathrm{dex}$ is possible owing to a combination of very weak solar lines, stronger lines in the globular giants, and strong hfs. The Pr II and Zr I absolute abundances are uncertain to 0.5 dex for similar reasons. The relative errors arising from the choice of atomic parameters in comparing globular giants in M3 and M13 must be significantly less, 0.07 dex even in the worst case.

\section{RESULTS OF THE ANALYSIS AND DISCUSSION OF ERRORS}

\section{a) General Remarks}

With the atomic parameters described in $\S$ III and the grid of model atmospheres of CFP, we can analyze the line spectrum of the giants in M3 and M13. The effective temperatures and surface gravities are taken from CFP, and the line spectrum is analyzed using the two closest models of the CFP grid. The choice of microturbulent velocity for the globular giants is specified by requiring the deduced abundance to be

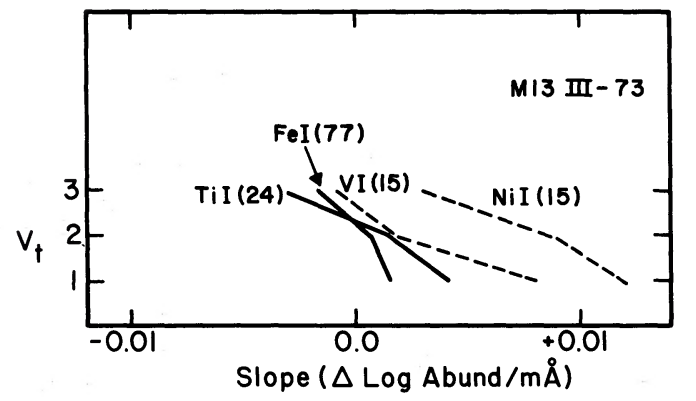

Fig. 1.-The slope of the least-squares straight-line fit to a plot of deduced abundances as a function of $W_{\lambda}$ for lines seen in M13 III-73 of Ti I, Fe I, VI, and Ni I is shown as a function of $V_{t}$. The model used had $T_{\text {eff }}=4200 \mathrm{~K}, \log g=1.0$, and $Z / Z_{\odot}=0.03$. The number of lines of each ion measured in this star is given in parentheses.

independent of $W_{\lambda}$ for ions with many measured lines (normally Ti I and Fe I). Figure 1 shows the slope of the least-squares fit to points representing all observed lines of an ion on a ( $W_{\lambda}$, abundance)-plot as a function of $V_{t}$. A value of $V_{t}=2.0 \mathrm{~km} \mathrm{~s}^{-1}$ (consistent with the values deduced [to an accuracy of $\pm 0.5 \mathrm{~km} \mathrm{~s}^{-1}$ ] from the plots similar to Fig. 1) was used for all the stars except M13 III-63, for which $1.5 \mathrm{~km} \mathrm{~s}^{-1}$ seemed required, and M13 IV-25, for which $2.2 \mathrm{~km} \mathrm{~s}^{-1}$ was used. This is in good agreement with the range of $V_{t}$ values previously found for metal-poor $\mathrm{K}$ giants (Sneden 1974; Gustafsson, Kjaergaard, and Anderson 1974).

The results of the abundance analysis using the two closest models of CFP are interpolated to the values of $T_{\text {eff }}$, surface gravity, metallicity, and $V_{t}$ listed in Table 3 to form the final abundance results listed in Tables 4A and 4B. The values of $\sigma$ listed for each ion in column (8) are typical (for the stars in each cluster) rms standard deviations for a single line calculated from the deduced abundances of the lines of each ion. Averages weighted by the individual weights for each line are given for ions with five or fewer lines, where the unweighted average had an rms deviation of more than 0.2 dex. The abundance for an ion with only one line is given in parentheses and was not used in the cluster averages, if the line had a weight less than $50 \%$ of the maximum weight for the star. We list in Table 5 the change in abundance for each ion that results from (a) a change in $T_{\text {eff }}$ by $+100 \mathrm{~K},(b)$ a change in $\log g$ by $0.50,(c)$ a change in metallicity of the model from the CFP grid from 0.03 to 0.01 times solar, $(d)$ an increase in $V_{t}$ from 2 to $3 \mathrm{~km} \mathrm{~s}^{-1}$, and (e) a decrease in $V_{t}$ from 2 to $1 \mathrm{~km} \mathrm{~s}^{-1}$, so that the reader can evaluate the effects of uncertainties in the temperature scale, mass, distance modulus, etc. The final results, listed in Tables $4 \mathrm{~A}$ and $4 \mathrm{~B}$, are the abundances for each ion for the atmospheric parameters of Table 3 , which contain the $T_{\text {eff }}{ }^{0}$ and $\log g^{0}$ for a mass of $0.8 M_{\odot}$ from CFP. The principal errors in the relative abundance determinations are those resulting from uncertainties in $V_{t}$ and in $T_{\text {eff }}$, and we 


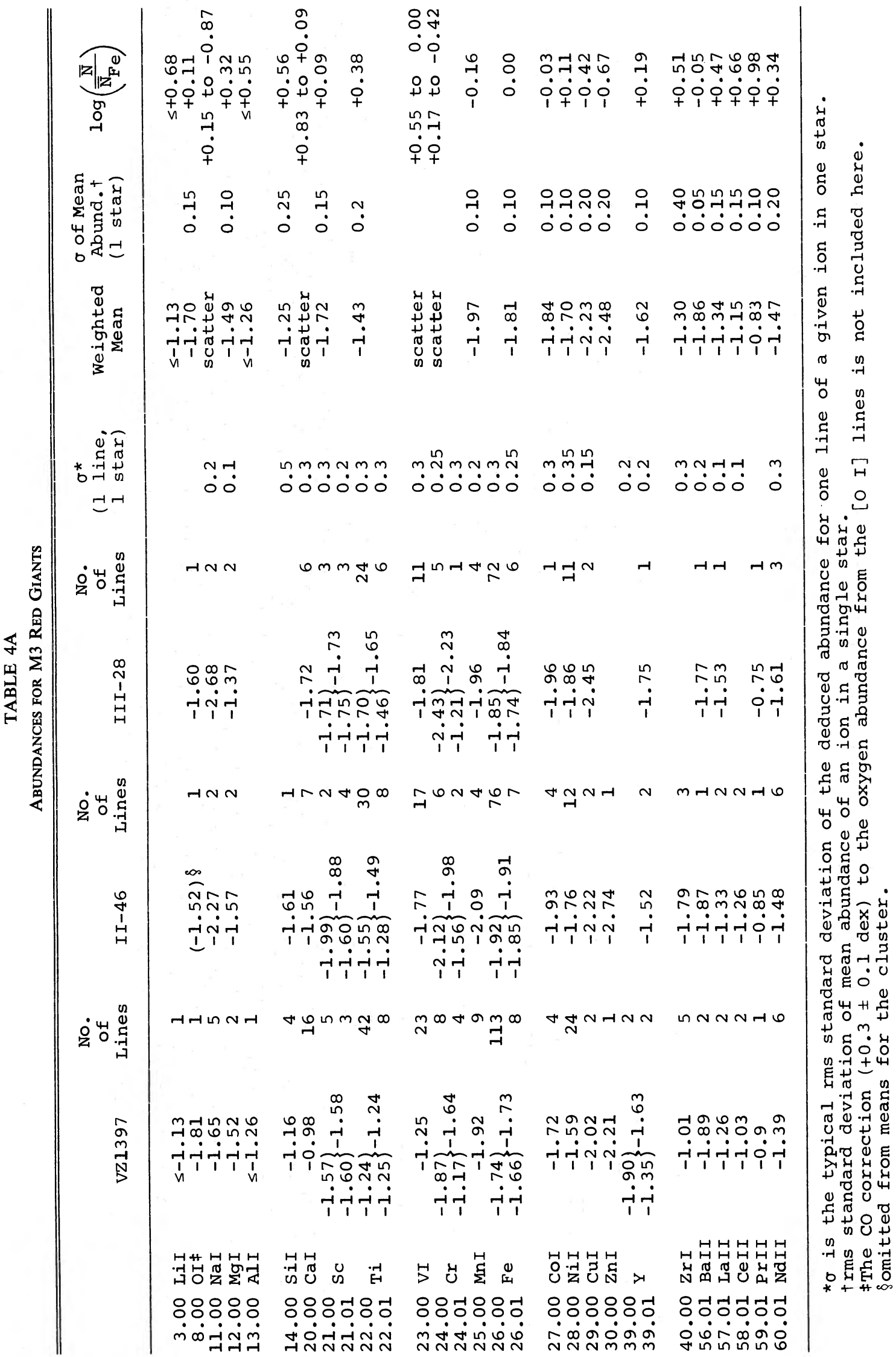




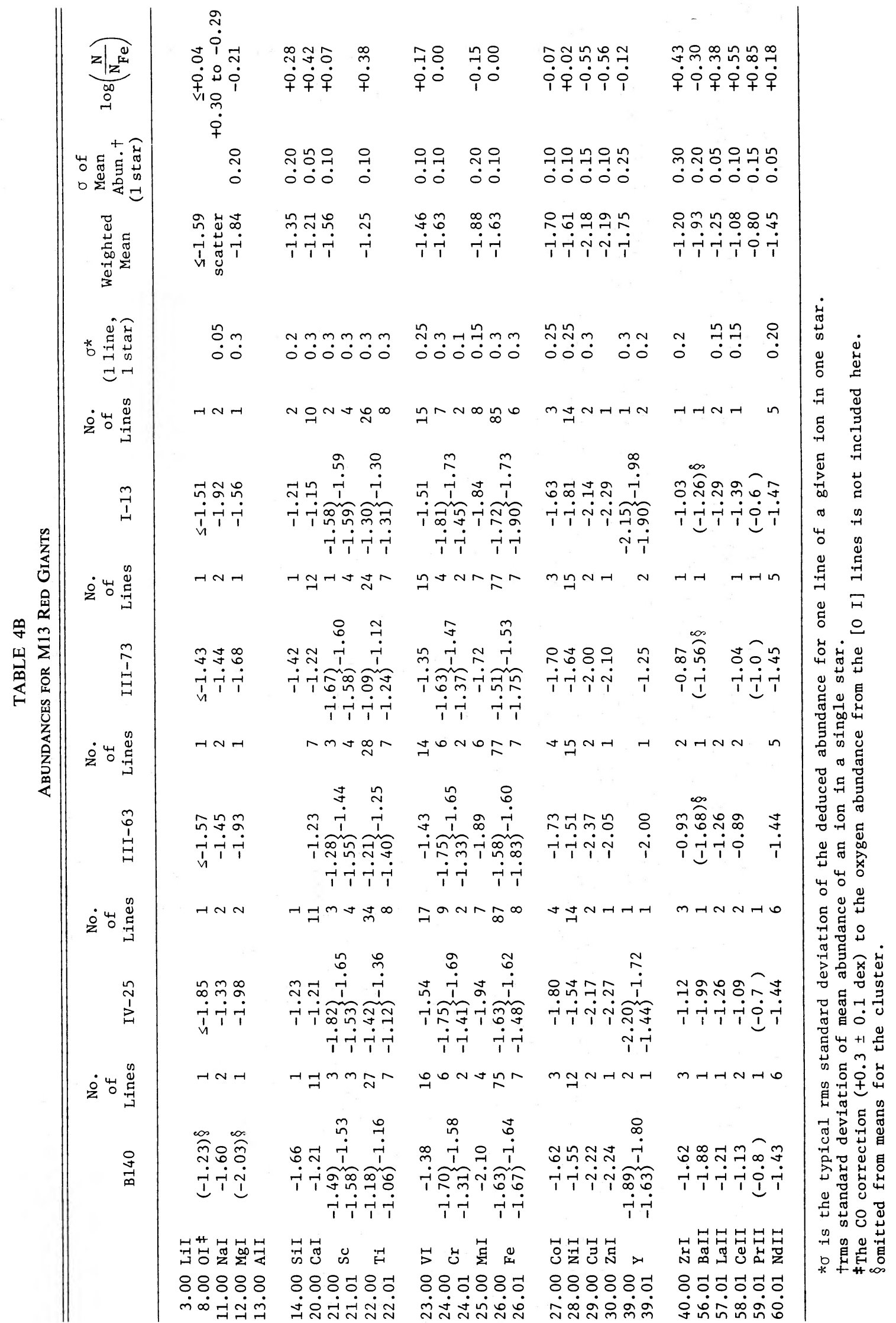


TABLE 5

Sensitivity of Abundances to Model Parameters

\begin{tabular}{|c|c|c|c|c|c|}
\hline \multirow[b]{2}{*}{ Ion } & \multicolumn{5}{|c|}{ Changes in Abundance $(\Delta \log N / H)$} \\
\hline & $\begin{array}{l}\Delta T \text { eff }= \\
+100 \mathrm{~K}\end{array}$ & $\begin{array}{c}\Delta \log g= \\
+0.5\end{array}$ & $\begin{array}{c}\Delta V_{t}=+1 \\
\left(\mathrm{~km}^{-1} \mathrm{~s}^{-1}\right)\end{array}$ & $\begin{array}{c}\Delta V_{t}=-1 \\
\left(\mathrm{~km}^{-1} \mathrm{~s}^{-1}\right)\end{array}$ & $\begin{array}{c}\text { Model } \\
Z=0.01\end{array}$ \\
\hline $\begin{array}{rl}3.00 & \mathrm{LiI} \\
8.00 \mathrm{OI} \\
11.00 \mathrm{NaI} \\
12.00 \mathrm{MgI} \\
13.00 \mathrm{AII}\end{array}$ & $\begin{array}{l}+0.27 \\
+0.03 \\
+0.21 \\
+0.07 \\
+0.10\end{array}$ & $\begin{array}{l}-0.02 \\
+0.21 \\
-0.14 \\
-0.09 \\
-0.02\end{array}$ & $\begin{array}{l}-0.02 \\
-0.01 \\
-0.13 \\
-0.06 \\
-0.02\end{array}$ & $\begin{array}{l}+0.03 \\
+0.02 \\
+0.04 \\
+0.10 \\
+0.03\end{array}$ & $\begin{array}{l}+0.14 \\
-0.19 \\
+0.07 \\
+0.01 \\
+0.05\end{array}$ \\
\hline $\begin{array}{l}14.00 \mathrm{SiI} \\
20.00 \mathrm{CaI} \\
21.00 \mathrm{ScI} \\
21.01 \mathrm{ScII} \\
22.00 \mathrm{TiI} \\
22.01 \mathrm{TiII}\end{array}$ & $\begin{array}{l}-0.05 \\
+0.14 \\
+0.22 \\
-0.02 \\
+0.20 \\
-0.04\end{array}$ & $\begin{array}{r}+0.11 \\
-0.08 \\
0.00 \\
+0.19 \\
-0.03 \\
+0.16\end{array}$ & $\begin{array}{l}-0.04 \\
-0.22 \\
-0.04 \\
-0.22 \\
-0.17 \\
-0.29\end{array}$ & $\begin{array}{l}+0.07 \\
+0.52 \\
+0.09 \\
+0.40 \\
+0.26 \\
+0.33\end{array}$ & $\begin{array}{l}-0.10 \\
+0.13 \\
+0.10 \\
-0.16 \\
+0.07 \\
-0.13\end{array}$ \\
\hline $\begin{array}{l}23.00 \mathrm{VI} \\
24.00 \mathrm{CrI} \\
24.01 \mathrm{CrII} \\
25.00 \mathrm{MnI} \\
26.00 \mathrm{FeI} \\
26.01 \mathrm{FeII}\end{array}$ & $\begin{array}{l}+0.21 \\
+0.17 \\
-0.09 \\
+0.13 \\
+0.08 \\
-0.13\end{array}$ & $\begin{array}{l}-0.02 \\
-0.03 \\
+0.20 \\
+0.01 \\
+0.04 \\
+0.25\end{array}$ & $\begin{array}{l}-0.15 \\
-0.14 \\
-0.06 \\
-0.03 \\
-0.27 \\
-0.22\end{array}$ & $\begin{array}{l}+0.33 \\
+0.17 \\
+0.13 \\
+0.05 \\
+0.35 \\
+0.26\end{array}$ & $\begin{array}{l}+0.07 \\
+0.08 \\
-0.12 \\
+0.07 \\
+0.02 \\
-0.16\end{array}$ \\
\hline $\begin{array}{l}27.00 \mathrm{COI} \\
28.00 \mathrm{NiI} \\
29.00 \mathrm{CuI} \\
30.00 \mathrm{ZnI} \\
39.00 \mathrm{YI} \\
39.01 \mathrm{YII}\end{array}$ & $\begin{array}{l}+0.09 \\
+0.06 \\
+0.10 \\
-0.07 \\
-0.15 \\
0.00\end{array}$ & $\begin{array}{l}+0.08 \\
+0.08 \\
+0.04 \\
+0.15 \\
-0.02 \\
+0.18\end{array}$ & $\begin{array}{l}-0.05 \\
-0.24 \\
-0.20 \\
-0.11 \\
-0.02 \\
-0.42\end{array}$ & $\begin{array}{l}+0.10 \\
+0.36 \\
+0.30 \\
+0.24 \\
+0.06 \\
+0.51\end{array}$ & $\begin{array}{l}-0.01 \\
-0.02 \\
+0.02 \\
-0.09 \\
+0.08 \\
-0.13\end{array}$ \\
\hline $\begin{array}{l}40.00 \mathrm{ZrI} \\
56.01 \mathrm{BaII} \\
57.01 \mathrm{LaII} \\
58.01 \mathrm{CeII} \\
59.01 \mathrm{PrII} \\
60.01 \text { NdII }\end{array}$ & $\begin{array}{r}+0.22 \\
+0.02 \\
+0.02 \\
0.00 \\
+0.02 \\
+0.01\end{array}$ & $\begin{array}{l}-0.01 \\
+0.19 \\
+0.19 \\
+0.19 \\
+0.19 \\
+0.21\end{array}$ & $\begin{array}{l}-0.05 \\
-0.30 \\
-0.02 \\
-0.02 \\
-0.02 \\
-0.02\end{array}$ & $\begin{array}{l}+0.15 \\
+0.62 \\
+0.10 \\
+0.04 \\
+0.05 \\
+0.15\end{array}$ & $\begin{array}{l}+0.06 \\
-0.14 \\
-0.17 \\
-0.16 \\
-0.16 \\
-0.16\end{array}$ \\
\hline
\end{tabular}

estimate these errors as \pm 0.2 dex when comparing abundances of various giants in Table 4 .

\section{b) Ionization Equilibrium}

The ionization equilibrium is an indication of the accuracy of the entire process, as the model atmosphere parameters were fixed from the infrared photometry of CFP. The best-determined case is Fe I versus $\mathrm{Fe}$ II. The largest difference between the deduced Fe I and $\mathrm{Fe}$ II abundances is $0.25 \mathrm{dex}$, and the mean difference is $-0.03 \mathrm{dex}$, while the mean of the absolute values of the differences is $0.12 \mathrm{dex}$. We consider this extremely satisfactory. The Sc ionization equilibrium is generally good, with differences less than $0.10 \mathrm{dex}$ in five of the stars. We ascribe the large differences (up to $0.40 \mathrm{dex}$ ) in the remaining stars to problems with the few observed Sc I lines, which tend to be weak and have hfs problems. The Ti ionization equilibrium is generally good, with differences of less than $0.2 \mathrm{dex}$ in five of the stars and a maximum discrepancy of 0.30 . The $\mathrm{Cr}$ and $\mathrm{Y}$ ionization equilibria are useless as tests of the accuracy of our analysis because of the few weak observed lines of $\mathrm{Cr}$ II and Y I. This generally good agreement with the Saha equation is especially pleasing in view of the difficulties other recent analyses of $\mathrm{K}$ stars (Mäckle et al. 1975b; Hearnshaw 1976) have encountered. We believe this good agreement to be due to the careful definition of the temperature scale for metal-poor $\mathrm{K}$ giants in CFP, the use of suitable model atmospheres including line opacities, and the knowledge of the reddening for these stars.

\section{c) Possible Non-LTE Effects}

There has been considerable controversy as to whether non-LTE effects cause overionization in cool, late-type giants such as we are considering. Auman and Woodrow (1975, hereafter AW) have predicted such an effect, and Kelch (1975) and Ramsey (1977) may have observational evidence that overionization occurs. The overionization is, however, predicted to be small for stars hotter than $4000 \mathrm{~K}$ and also will not affect the electron pressure (and hence the model atmosphere itself) for $T_{\text {eff }}$ greater than $3500 \mathrm{~K}$. Because our $\mathrm{Fe}$ ionization equilibrium (i.e., difference in the deduced abundance of $\mathrm{Fe} I$ and $\mathrm{Fe}$ II) is very good for the $T_{\text {eff }}$ and surface gravity prescribed from observations by CFP, an overionization of Fe larger than a factor of 1.5 is not possible. The deduced abundances of $\mathrm{O}$ I, Ba II, $\mathrm{Zn} \mathrm{I}$, and the rare earths must be correct to within 0.05 dex, as the ions observed represent over $98 \%$ of the total for those elements. The $\mathrm{Ca}, \mathrm{Mg}, \mathrm{V}$, and $\mathrm{Na}$ abundances, deduced only from lines of the neutral species where most of those ions are singly ionized, could be significantly affected, with a predicted effect (AW) for $T_{\text {eff }}=4000 \mathrm{~K}$ that is a factor of 10 for $\mathrm{Ca}$ and $\mathrm{Na}$ and is negligible for $\mathrm{Mg}$ (AW consider only the elements $\mathrm{Mg}, \mathrm{Ca}, \mathrm{Al}, \mathrm{Na}$, and $\mathrm{K})$. AW do not have atomic or molecular line blanketing included in their models and estimate by comparing observed and computed ultraviolet fluxes that their predicted overionizations are too large by a factor of approximately 10 , which would make the predicted effect for $\mathrm{K}$ giants insignificant. However, the surface 
gravity of their $4000 \mathrm{~K}$ model is significantly higher than that of the globular cluster giants, and their models are for solar abundances; both act to underestimate the magnitude of the non-LTE overionizations for the globular cluster giants. To obtain an estimate of the maximum possible overionization of $\mathrm{Ca}$ and $\mathrm{Na}$, we determine (following $\mathrm{AW}$ ) for $\mathrm{Ca}$ and $\mathrm{Na}$

$$
\frac{\left(N_{a} / N_{1}\right)_{\text {non-LTE }}}{\left(N_{2} / N_{1}\right)_{\text {LTE }}}=\frac{J_{v_{1}}(\bar{\tau})}{B_{v_{1}}(\bar{\tau})},
$$

where $\nu_{1}$ is the limit of the photoionization continuum of the first excited state of $\mathrm{Ca}$ I or $\mathrm{Na} I, \bar{\tau}$ is the mean depth of formation of the observed lines of the ion, $B$ is the Planck function, $J$ is the mean value of the intensity averaged over solid angle, $N_{2}$ is the number of ionized atoms, while $N_{1}$ is the number of neutral atoms of a given element. These ratios have been determined, using two representative models from the CFP grid, for the $\nu_{1}$ appropriate to $\mathrm{Ca}$ I and $\mathrm{Na}$, and are 2-3 over the entire line-forming region, approaching 1 only deep in the atmosphere. However, again following $\mathrm{AW}$, we must apply a correction, because the ultraviolet fluxes predicted by our model atmospheres are too large (i.e., we are missing some ultraviolet opacity). This correction is a factor of 1.4 , based on the data of CFP; the correction factor is itself underestimated, because the missing opacity must be due to absorption rather than scattering. Therefore, we can confidently assert that the maximum possible overionization of $\mathrm{Ca}$ and $\mathrm{Na}$ is a factor of 2 . It appears from the model calculations that, over the restricted range of $T_{\text {eff }}, \log g$, and $Z$ represented by the globular giants discussed here, if the overionization is actually present, it is constant in all the stars (i.e., $J_{v_{1}} / B_{v_{1}}$ is constant). We urge theoreticians to explore this problem in more detail for other ions, particularly V I, Ti I, $\mathrm{Cr}$, and Sc I, using line-blanketed model atmospheres.

\section{d) Discussion of Final Abundances}

The final mean abundances for five stars in M13 and three in M3 are plotted in Figure 2 as a function of atomic number. Elements with a scatter from star to star greater than 0.3 dex are plotted as a vertical bar extending over the range of abundances observed in the stars for that cluster, while adjacent atomic numbers with good determinations are connected by a straight line. Particularly uncertain values for $\mathrm{Zr}$ I and Pr II are enclosed in a circle.

We first discuss the general pattern of the abundances. Oxygen is not overabundant with respect to Fe by more than 0.2 dex. However, the oxygen abundance must be corrected for oxygen tied up in $\mathrm{CO}$. Based on calculations of molecular equilibria using the code of Kurucz (1970), the estimated correction is $0.3 \pm 0.1$ dex in the oxygen abundance deduced from atomic oxygen only. From $\mathrm{Ca}$ to $\mathrm{Zn}$ (atomic numbers 20-30), the abundance of every element has been determined. There is some evidence for an overdeficiency of 0.3 dex of the odd elements, compared with the adjacent even elements-particularly Sc, Mn, and $\mathrm{Cu}$ (see Fig. 2)-although the overdeficiencies are smaller than those found by Peterson (1976a) in four

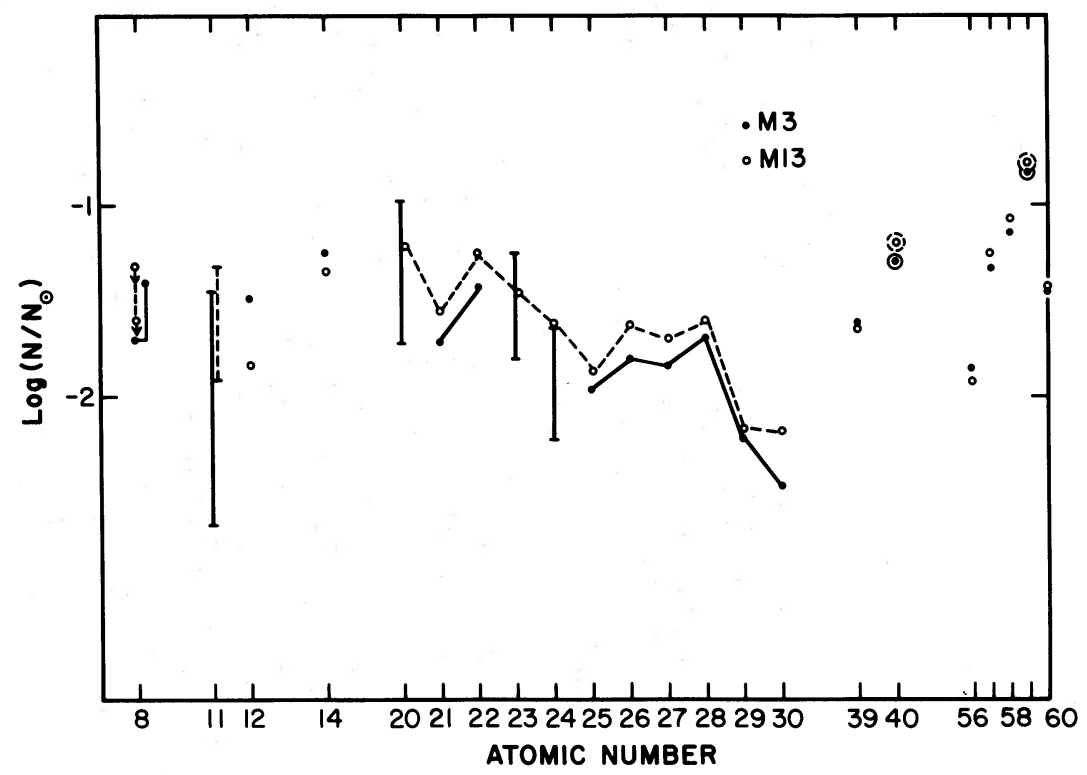

Fig. 2.-The abundance with respect to the Sun is shown for elements in M3 and M13 as a function of atomic number. Particularly uncertain values are circled. A vertical bar indicates the range of abundances (greater than the errors expected from the analysis) found for that element in stars of a given cluster. The $\mathrm{V}$ and $\mathrm{Cr}$ abundance range for the $\mathrm{M} 3$ stars can be reduced to an acceptable level by a small temperature shift as discussed in $\S$ IV $d$. The M13 abundances are denoted by open circles and dashed lines; the M3 results are indicated as filled circles and solid lines. The correction to the $\mathrm{O}$ abundance for $\mathrm{CO}$ formation is indicated by the upward-shifted $O$ points. 
field metal-poor dwarfs. The even iron-peak nuclei $\mathrm{Cr}, \mathrm{Fe}$, and $\mathrm{Ni}$ all have the same deficiency with respect to the Sun, while Ca and Ti are overabundant by a factor of 2 with respect to $\mathrm{Fe}$. The light nuclei $\mathrm{Na}$, $\mathrm{Mg}$, and $\mathrm{Si}$ have deficiencies that lie between the $\mathrm{Ca}$ and $\mathrm{Fe}$ deficiencies. $\mathrm{Cu}$ and $\mathrm{Zn}$ are significantly more deficient than the mean even iron-peak elements. Ba shows a deficiency with respect to Fe of -0.2 dex, while $\mathrm{Y}$ has an abundance equal to that of $\mathrm{Fe}$. It is rather surprising that the elements past $\mathrm{Ba}$-in particular, Nd II (which is the best-determined case)are not overdeficient and indeed appear to be slightly overabundant with respect to $\mathrm{Fe}$. These general trends are seen in both clusters, and it is hoped that the forthcoming results from analysis of stars in the more metalpoor globulars will provide an interesting comparison.

The odd-even effect for $\mathrm{Na}, \mathrm{Al}, \mathrm{V}, \mathrm{Mn}$, and $\mathrm{Co}$ is a prediction of explosive burning of $\mathrm{O}$ and $\mathrm{Si}$ in single stars (Arnett 1971), but there is no evidence for an overdeficiency of $\mathrm{Na}$ larger than $0.3 \mathrm{dex}$ in previous analyses of metal-poor stars (Pagel 1970; Wolffram 1972). $\mathrm{Cu}$ and $\mathrm{Zn}$ might be expected to be overdeficient, as they are generally believed to be among the heaviest elements produced by explosive carbon burning and by nuclear statistical equilibrium near the iron peak (Cameron 1973), although Zn was found to be slightly overabundant with respect to $\mathrm{Fe}$ in the extremely metal-poor giant HD 122563 (Wolffram 1972). The overdeficiency of only the heavy $s$-process element $\mathrm{Ba}$ and the absence of an overdeficiency for the lighter $s$-process element Y represent common phenomenon in metal-poor stars of intermediate and extreme metal deficiency (Pagel 1970), and are related to the scarcity of seed nuclei and to how far the $s$ process has proceeded in previous generations of stars. The general overabundance of the elements heavier than $\mathrm{Ba}$ is surprising. Although previous evidence of this point is rather limited, Spite and Spite (1973) encountered a similar effect, which seems contrary to the $s$-process calculations of Seeger, Fowler, and Clayton (1965) and others. It is, of course, possible, although improbable, that the isotopic distribution among these elements is quite different from that of the solar system, so that the $r$-process nuclei could be quite abundant and the $s$-process nuclei could show the expected increasing overdeficiency for heavier nuclei. The slight overabundance of $\mathrm{Ca}$ and $\mathrm{Ti}$ is also in agreement with previous results (Pagel 1970; Wolffram 1972) for metal-poor field stars. Hearnshaw (1975) has summarized the limited evidence, stating that, for metal-poor halo stars, $\mathrm{C} / \mathrm{Fe}$ remains constant for dwarfs. Our results indicate that, in M3, $\mathrm{O} / \mathrm{Fe}$ (including the $\mathrm{CO}$ correction) is $+0.4 \pm 0.2$, which is close to the value deduced for a few metalpoor field giants by Lambert, Sneden, and Ries (1974) and by Conti et al. (1967). It does not appear probable, both from theoretical models and from observations of solar metallicity stars (Lambert and Ries 1977), that $\mathrm{O}$ is significantly affected by mixing. Therefore, the high $\mathrm{O}$ abundance for the $\mathrm{M} 3$ stars must be primordial.

We now consider the difference between the mean
M3 and M13 abundances. A perusal of Figure 2 shows clearly that M13 is more metal-rich than M3 by 0.2 dex in the region of atomic numbers 20-28. However, for the lighter elements-particularly $\mathrm{Mg}$ and $\mathrm{Si}-\mathrm{M} 13$ is marginally more metal-poor by perhaps 0.15 dex. One must recall that the relative abundances presented here are extremely accurate even though there may be some problems with the absolute abundances. Narrowband infrared photometry of CO for stars in M3 and M13 by CFP indicates that the M3 giants have more $\mathrm{CO}$ than do those of $\mathrm{M} 13$, in agreement with the reversal of abundance ordering of the two clusters that has been noted for the light elements as compared with the iron peak. The heavier elements past the iron peak are identical in the two clusters to within the errors.

One of the most interesting questions resulting from an attempt to explain the abundance distribution is the cause of variation in abundance seen for some elements between stars in the same cluster. The elements with scatter are: $\mathrm{Na}$ I only for M13; $\mathrm{Na}$, $\mathrm{Ca} \mathrm{I}, \mathrm{V}$ I, and $\mathrm{Cr}$ I for M3. Let us first explore the hypothesis that the scatter is caused by errors in the temperature scale. For M3, where the scatter is prominent, the stars seem divided into two groups. M3 II-46 and M3 III-28 are in the more metal-poor group, with $\mathrm{Na}, \mathrm{Ca}, \mathrm{V}$, and $\mathrm{Cr}$ abundances lower by 0.4-0.6 dex. VZ 1397 is the star most metal-rich in all of these elements. A temperature error of $300 \mathrm{~K}$ for VZ 1397, based on Table 4, is required to produce this spread. However, $\mathrm{Ti}$ I and $\mathrm{Sc}$ I are as temperature sensitive as $\mathrm{Ca}$ I, Cr I, and VI, and they show much smaller scatter among the M3 stars, although it is interesting to note that both Ti and Sc are more abundant in VZ 1397 than in the other M3 stars by 0.3 dex. Mn I is as temperature sensitive as $\mathrm{Ca}$ I (see Table 5), and yet the total range of $\mathrm{Mn}$ abundances in the M3 stars is only 0.17 dex. Furthermore, the Fe ionization equilibrium is good, and the $\mathrm{Ti}$ ionization equilibrium suggests a maximum temperature error of $100 \mathrm{~K}$ in the sense that II-46 and III-28 should perhaps be hotter by $100 \mathrm{~K}$. This will reduce to acceptable levels the scatter in $\mathrm{Cr}$ and $\mathrm{V}$ abundances and will make $\mathrm{Ti}$ and $\mathrm{Sc}$ more constant for the three stars in M3, but it fails to eliminate the problem of high scatter in $\mathrm{Ca}^{1}$ and $\mathrm{Na}$ abundances. We therefore rule out errors in the temperature scale as the cause of the deduced scatter for $\mathrm{Ca}$ and $\mathrm{Na}$. A similar perusal of the appropriate columns in Table 5 can rule out errors in $V_{t}$ as a cause of the scatter. Assuming that our choice of $T_{\text {eff }}$ and $V_{t}$ is essentially correct, relative errors in the abundance analysis procedure discussed above are much smaller than the observed scatter and possible non-LTE effects are also too small. The lines involved are too weak (with the

\footnotetext{
${ }^{1}$ If only the seven $\mathrm{Ca}$ I lines measured in M3 II-46 are used for VZ 1397, the resulting $\mathrm{Ca}$ abundance in VZ 1397 is reduced 0.3 dex below that given in Table $4 \mathrm{~A}$. It is unfortunate that the $\mathrm{Ca}$ I lines near $6160 \AA$ were measured on only one spectrum of VZ 1397 and not at all in the other M3 stars. It is thus perhaps marginally possible to explain the range in deduced $\mathrm{Ca}$ abundance for the M3 stars as a temperature error, if one rejects the nine uncertain $\mathrm{Ca} I$ lines in VZ 1397.
} 
possible exception of those of $\mathrm{Na} \mathrm{I}$ ), and there is not enough molecular opacity for a surface-cooling effect such as that advocated by Peterson (1976b) to be relevant.

We therefore conclude that the scatter of $\mathrm{Na}$ I and possibly Ca I shown in M3 is real, as perhaps is the scatter of only $\mathrm{Na}$ I observed in M13. We note that Freeman and Rodgers (1975) have observed a large spread in $\mathrm{Ca}$ abundance for the RR Lyrae stars in $\omega$ Cen, although in $\omega$ Cen the excess width of the giant branch (Cannon and Stobie 1973) provides additional evidence for star-to-star abundance variations. We recall that VZ 1397-relative to the other two M3 stars-has excess $\mathrm{Na}$ and $\mathrm{Ca}$, with smaller excesses of $\mathrm{V}, \mathrm{Cr}, \mathrm{Sc}$, and $\mathrm{Ti}$ that are probably due to a $100 \mathrm{~K}$ error in the temperature scale. The most reliable, very heavy elements ( $\mathrm{Y}, \mathrm{Ba}$, and $\mathrm{Nd})$ are identical for all three M3 stars. Can this be a result of convective mixing? While CNO and s-process anomalies are related to mixing phenomena, there is no way to affect the abundances of $\mathrm{Na}$ and $\mathrm{Ca}$ through mixing (Demarque and Mengel 1971; Sackmann, Smith, and Despain 1974; Dearborn, Bolton, and Eggleton 1975). Furthermore, the CH stars, which are Population II carbon stars that have definitely undergone mixing (Wallerstein and Greenstein 1964), show excesses of C and of the heavy elements past $\mathrm{Zr}$, but have $\mathrm{Ca} / \mathrm{Fe}$ that is normal and $\mathrm{Na} / \mathrm{Fe}$ that is less than solar by -0.5 dex. $\mathrm{CH}$ stars and stars with enhanced $\mathrm{Ba}$ and Sr have been found in $\omega$ Cen (Dickens and Bell 1976). Although it is tempting to explain the unexpectedly large abundances of the heavy elements past $\mathrm{Zr}$ in the M3 and M13 red giants as a result of a similar mixing process, this does not solve the problem of the observed scatter of $\mathrm{Na}$ and $\mathrm{Ca}$ in the M3 stars. Mixing is also psychologically unsatisfactory, as one would then be forced to postulate that it occurred on a much larger scale in M3 than in M13, and these clusters seem to be similar in most respects.

We must therefore conclude that the scatter in the M3 stars was present in the gas from which the giants condensed. Either there was some time spread in star formation so that, before the younger star-which must be VZ1397-was formed, there were one or more extra supernovae, or all the stars are coeval but the supernova ejecta did not mix uniformly throughout the gas cloud from which the M3 stars condensed.

Calculations for explosive nucleosynthesis in metalpoor stars (Truran and Arnett 1971) indicate that production of large amounts of $\mathrm{O}, \mathrm{Ne}, \mathrm{Mg}, \mathrm{S}, \mathrm{Ar}$, and $\mathrm{Ca}$ may be expected from a single supernova. Unfortunately, $\mathrm{Na}$ is not expected to be produced, nor is it possible to reduce the amount of $\mathrm{O}$ produced. Cas A, a Type II supernova remnant, has a large $\mathrm{O}$ excess (Peimbert and van den Bergh 1971). While the $\mathrm{O}$ abundances for M3 may be wrong, the scatter in $\mathrm{Na}$ is real. It seems, therefore, that a single supernova or a few extra supernovae will not explain the anomalies of VZ 1397 with respect to the other M3 stars. We are thus left with a most unsatisfying explanation of inhomogeneous mixing of previous generations of supernova ejecta in the gas cloud from which M3 condensed.

If we regard the lower abundances of the M3 stars (i.e., excluding VZ 1397) as representative of the initial gas of M3 and use the lower Na I results for M13, then the predictions of explosive nucleosynthesis in single, metal-deficient stars, as described by Truran and Arnett (1971), seem capable of producing the observed abundance patterns through the iron-peak elements in both clusters. It will be of great interest to extend this analysis to M15 and M92.

\section{SUMMARY OF CONCLUSIONS}

Using high-dispersion $4 \mathrm{~m}$ echelle spectra, an abundance analysis has been performed for five stars in the globular cluster M13 and three in M3. One of the most encouraging aspects of this analysis is that, in spite of problems with $g f$-values, damping constants, and hyperfine structure, the results are internally consistent; the ionization equilibrium is reasonable at the temperatures determined from infrared photometry by CFP and at the surface gravities required for the known distance moduli of the clusters assuming a mass of $0.8 M_{\odot}$. Furthermore, for most elements, all the stars in a given cluster have the same abundances to within the errors of the determinations. A summary of the important results is listed below.

1 . The $[\mathrm{O} / \mathrm{Fe}]$ ratio is $+0.4 \pm 0.2$ dex larger than solar in M3 and less than +0.4 dex in M13.

2. Some indication of a larger overdeficiency of odd- $Z$ elements is seen, particularly of Sc, $\mathrm{Mn}$, and $\mathrm{Cu}$, as predicted by explosive nucleosynthesis in single, metal-poor stars (Arnett 1971).

3. The mean Fe deficiency is -1.8 dex for M3 and -1.6 dex for M13, while M3 is marginally more metal-rich in $\mathrm{Mg}$ and $\mathrm{Si}$. This crossover of deficiencies in the light versus heavy elements, for these two clusters is given further observational support by the narrow-band CO photometry of CFP. If this crossover between the M3 and M13 abundance deficiencies is not a result of mixing, then it has profound implications for the theory of the chemical history of the Galaxy. A different mass distribution of previous generations of stars in M3 as opposed to those in M13 would be required to produce the observed effect.

4. In both clusters $\mathrm{Ba}$ is slightly overdeficient, but the elements heavier than $\mathrm{Ba}$ are not.

5. $\mathrm{Ca}$ and $\mathrm{Ti}$ are slightly overabundant $(0.3 \mathrm{dex})$, while the even iron-peak nuclei $(\mathrm{Cr}, \mathrm{Fe}, \mathrm{Ni})$ have the same deficiency.

6. The manner in which the abundance of $\mathrm{Na}$ in M13 and of Na and $\mathrm{Ca}$ in M3 appears to vary from star to star indicates that the possible errors in the analysis do not produce all of the observed scatter. Convective mixing, as theoretically understood and as seen in the weak G-band stars (Norris and Zinn 1977) and the CH stars (Wallerstein and Greenstein 1964), cannot produce the observed scatter. One can also eliminate the possibility that the stars in M3 are not 
coeval and that there were a few extra supernovae during the time interval in which the stars formed, since $\mathrm{Na}$ is not expected to be significantly produced in supernovae in metal-poor stars (Truran and Arnett 1971). The only viable explanation, although a most unsatisfactory one, is incomplete mixing of supernova ejecta through the gas cloud out of which M3 con- densed. We hope that the forthcoming analysis of the M92 and M15 red giants will confirm these trends.

We are grateful to Mr. Ed Carder of KPNO for tracing all the spectra with the PDS microphotometer. We thank Dr. R. L. Kurucz for permission to use his computer programs.

\section{APPENDIX}

\section{RADIAL VELOCITIES, INTERSTELLAR LINES, AND H $\alpha$ PROFILES}

Radial velocities were measured for all the stars using only the night-sky line at $5577 \AA$, as the Th-Ar comparison spectrum was not well exposed on these plates. In Table 6 we list the mean heliocentric radial velocities for all the stars. In only one case did any of the measured velocities differ by more than $10 \mathrm{~km} \mathrm{~s}^{-1}$ from the mean velocity; this was for M3 III-28, where one plate gave a value of $-148 \mathrm{~km} \mathrm{~s}^{-1}-15 \mathrm{~km} \mathrm{~s}^{-1}$ lower than the mean. Therefore, all the stars are members and there is no evidence of any velocity variation with a semiamplitude greater than $15 \mathrm{~km} \mathrm{~s}^{-1}$.

Some details of the stellar $\mathrm{H} \alpha$ profiles are also listed in Table 6, as they are useful for estimates of mass-loss rates (Cohen 1976b). We list $W_{\lambda}$ for the absorption component of $\mathrm{H} \alpha$ and indicate whether emission is present in the wings of $\mathrm{H} \alpha$ and, if so, in which wings. If emission is indicated as dubiously present, it was not seen on all the plates of a given star. The full width at half-maximum (FWHM) of the $\mathrm{H} \alpha$ absorption is 1.1-1.25 $\AA$. These stars do not obey the luminosity-FWHM(H $\alpha$ ) relationship of Kraft, Preston, and Wolff (1964).

The last columns of Table 6 list the mean equivalent widths and heliocentric radial velocities for the interstellar components of $\mathrm{Na}$ I. No interstellar components were seen at velocities between $-30 \mathrm{~km} \mathrm{~s}^{-1}$ and the $V_{r}$ of the globular cluster, although components with a $V_{r}$ within $25 \mathrm{~km} \mathrm{~s}^{-1}$ of the stellar velocity would be lost in the stellar D lines. There were no interstellar components detected toward M3, in agreement with the 0.00 mag color excess for this cluster determined by Sandage (1970). For M13, two components are present at -25 and $-11 \mathrm{~km} \mathrm{~s}^{-1}$. Since these components have velocities close to zero, and since Cohen (1974) and Cohen and Meloy (1975) have demonstrated that the halo gas has an abnormally large ratio, $N(\mathrm{Ca}$ II) $/ N(\mathrm{Na}$ I), we may safely assume that these are two local clouds within the galactic plane. This gives us a unique opportunity to compare Na I column densities through two clouds (and only two clouds) with unusually high spatial resolution. If we assume a $150 \mathrm{pc}$ scale height in $Z$ for the galactic plane, then the clouds must be within $230 \mathrm{pc}$ of the Sun. Cloud A appears to be uniform over the southern part of the cluster at a column density for $\mathrm{Na}$ I of $3 \times 10^{11}$ atoms $\mathrm{cm}^{-2}$. The column density increases toward the northern part of the cluster to $1 \times 10^{12}$ atoms $\mathrm{cm}^{-2}$. This increase occurs within $5^{\prime}$, or less than $0.3 \mathrm{pc}$. Cloud B is seen in only two of the stars. Assuming that these two clouds have the normal Na $\mathbf{I} / \mathbf{H}$ I for clouds in the plane given by Cohen (1975), the reddening to M13 is 0.025 mag, and it may be slightly variable over the cluster. Sandage (1970) obtained $E_{B-V}=0.03$ mag.

TABLE 6

$V_{r}$, Interstellar Na Components, and H $\alpha$ Profile Data

\begin{tabular}{|c|c|c|c|c|c|c|c|c|c|}
\hline \multirow[b]{3}{*}{ Star } & \multirow{3}{*}{$\begin{array}{c}V_{r} \\
\mathrm{~s}^{-1}\end{array}$} & \multirow{2}{*}{\multicolumn{2}{|c|}{$\mathrm{H} \alpha$ Data }} & \multicolumn{6}{|c|}{ Interstellar $\mathrm{Na}$} \\
\hline & & & & $\overline{W_{\lambda}(5889)}$ & $\frac{\text { Component } 1}{W_{\lambda}(5895)}$ & $V_{r}$ & $\overline{W_{\lambda}(5889)}$ & omponent 2 & $V_{r}$ \\
\hline & & (A) & Emission & $\mathrm{m} \AA$ & $\mathrm{m} \AA$ & $\mathrm{km} \mathrm{s}^{-1}$ & $\mathrm{~m} \AA$ & $\mathrm{m} \AA$ & $\mathrm{km} \mathrm{s}^{-1}$ \\
\hline \multicolumn{10}{|l|}{ M13 } \\
\hline B140 & -276 & 0.85 & \multirow{5}{*}{$\begin{array}{c}\text { blue } \\
\text { red \& blue } \\
\text { no } \\
\text { blue? } \\
\text { red \& blue }\end{array}$} & 65 & \multirow{5}{*}{$\begin{array}{l}57 \\
28 \\
86 \\
27\end{array}$} & \multirow{5}{*}{$\begin{array}{l}-24 \\
-25 \\
-30 \\
-26\end{array}$} & & & \\
\hline IV -25 & -263 & 1.00 & & 48 & & & & & \\
\hline$I-13$ & -253 & 1.18 & & 121 & & & $a$ & $a$ & -10 \\
\hline III -63 & -260 & 0.91 & & 51 & & & & & \\
\hline III -73 & -255 & 1.02 & & & & & 97 & 50 & -13 \\
\hline \multicolumn{10}{|l|}{ M3 } \\
\hline Vz 1397 & -162 & 0.97 & blue? & none & & & & & \\
\hline III-28 & -163 & 1.03 & blue? & none & & & & & \\
\hline II -46 & -167 & 1.02 & no & none & & & & & \\
\hline
\end{tabular}

a - weak component, about $1 / 3$ as strong as principal component 


\section{REFERENCES}

Arnett, W. D. 1971, Ap. J., 166, 153.

Auman, J. R., and Woodrow, J. E. J. 1975, Ap. J., 197, 163 (AW).

Brault, J., and Testerman, L. 1977, unpublished.

Bridges, J. M., and Wiese, W. L. 1970, Ap. J. (Letters), 161, L71.

Cameron, A. G. W. 1973, Space Sci. Rev., 15, 121.

Cannon, R. D., and Stobie, R. S. 1973, M.N.R.A.S., 162, 207.

Cohen, J. G. 1974, Ap. J., 194, 37. 1975, Ap. J., 197, 117.

p. 8 .

1976a, KPNO-CTIO Quart. Bull., July-September,

p. 8. 1976b, Ap. J. (Letters), 203, L127.

Cohen, J. G., Frogel, J. A., and Persson, S. E. 1978, Ap. J., 222, 165 (CFP).

Cohen, J. G., and Meloy, D. A. 1975, Ap. J., 198, 545.

Conti, P. S., Greenstein, D. L., Spinrad, H., Wallerstein, G., and Vardya, M. A. 1967, Ap. J., 148, 105.

Corliss, C. H., and Bozman, W. R. 1962, NBS Monog., No. 53 (CB).

Dearborn, D. S., Bolton, A. F. C., and Eggleton, P. P. 1975, M.N.R.A.S., 170, 7P.

Demarque, P., and Mengel, J. G. 1971, Ap. J., 164, 317.

Dickens, R. J., and Bell, R. A. 1976, Ap. J., 207, 506.

Foy, R. 1972, Astr. Ap., 18, 26.

Freeman, K. C., and Rodgers, A. W. 1975, Ap. J. (Letters), 201, L71.

Frogel, J. A., and Persson, S. E. 1977, private communication.

Furenlid, I., and Condal, A. R. 1976, preprint.

Gingerich, O., Noyes, R. W., Kalkofen, W., and Cuny, Y. 1971, Solar Phys., 18, 347.

Griffin, R. 1969, M.N.R.A.S., 143, 381.

Griffin, R. F. 1968, A Photometric Atlas of the Spectrum of Arcturus (Cambridge, England: Cambridge Philosophical Society).

Gustafsson, B., Kjaergaard, P., and Anderson, S. 1974, Astr. Ap., 34, 99.

Hearnshaw, J. B. 1972, Mem. R.A.S., 77, 55

-. 1975, Astr. Ap., 38, 271. 1976, Astr. Ap., 51, 71.

Helfer, H. L., Wallerstein, G., and Greenstein, J. L. 1959, Ap. J., 129, 700.

Hogg, H. S. 1973, Pub. David Dunlap Obs., Vol. 3, No. 63.

Holweger, H. 1971, Astr. Ap., 10, 128.
Holweger, H. 1972, Solar Phys., 25, 14.

Holweger, H., and Oertel, K. B. 1971, Astr. Ap., 10, 434.

Kelch, W. L. 1975, Ap. J., 195, 679.

Kraft, R. P., Carbon, D., and Butler, D. 1978, in preparation.

Kraft, R. P., Preston, G. W., and Wolff, S. C. 1964, Ap. J., 140, 235.

Kurucz, R. L. 1970, Smithsonian Ap. Obs. Spec. Rept., No. 309. 1978 , in preparation.

Kurucz, R. L., and Peytremann, E. 1975, Smithsonian Ap. Obs. Spec. Rept., No. 362.

Kurucz, R. L., Peytremann, E., and Avrett, E. H. 1974, Blanketed Model Atmospheres for Early-Type Stars (Washington: Smithsonian Institution Press).

Lambert, D. L., and Ries, L. M. 1977, Ap. J., 217, 508.

Lambert, D. L., Sneden, C., and Ries, L. M. 1974, Ap. J., 188, 97.

Mäckle, R., Griffin, R., Griffin, R., and Holweger, H. 1975a, Astr. Ap. Suppl., 19, 303.

Mäckle, R., Holweger, H., Griffin, R., and Griffin, R. 1975b, Astr. Ap., 38, 239.

Moore, C. E., Minnaert, M. G. J., and Houtgast, J. 1966, NBS Monogr., No. 61 (MMH).

Norris, J., and Zinn, R. 1977, Ap. J., 215, 174.

Pagel, B. E. J. 1970, Quart.J.R.A.S., 11, 172.

Peimbert, M., and van den Bergh, S. 1971, Ap. J., 167, 223.

Peterson, R. 1976a, Ap. J., 206, 800.

-1976b, Ap. J. Suppl., 30, 61.

-. 1978, Ap. J., 222, 181.

Ramsey, L. W. 1977, $A p . J ., 215,827$.

Sackmann, I. J., Smith, R. L., and Despain, K. H. 1974, Ap. J., $187,555$.

Sandage, A. R. 1970, Ap. J., 162, 841.

Seeger, P. A., Fowler, W. A., and Clayton, D. D. 1965, Ap. J. Suppl., 11, 121.

Smith, M. A., Testerman, L., and Evans, J. C. 1976, Ap. J., 207, 308.

Sneden, C. 1974, Ap. J., 189, 493.

Spite, M., and Spite, F. 1973, Astr. Ap., 23, 63.

Truran, J. W., and Arnett, W. D. 1971, Ap. Space Sci., 11, 430.

Wallerstein, G., and Greenstein, J. G. 1964, Ap. J., 139, 1163.

Wiese, W. L., and Fuhr, J. R. 1975, J. Phys. Chem. Ref. Data, 4, 263.

Wolffram, W. 1972, Astr. Ap., 17, 17.

Judith G. CohEn: Kitt Peak National Observatory, P.O. Box 26732, Tucson, AZ 85726 